\title{
Perceptual Decision-Making: Biases in Post-Error Reaction Times Explained by Attractor Network Dynamics
}

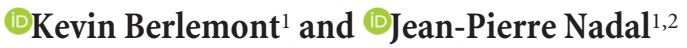 \\ ${ }^{1}$ Laboratoire de Physique Statistique, École Normale Supérieure, PSL University, Université Paris Diderot, Université Sorbonne Paris Cité, Sorbonne \\ Université, CNRS, 75005 Paris, France and 2Centre d'Analyse et de Mathématique Sociales, École des Hautes Études en Sciences Sociales, PSL University, \\ CNRS, 75006 Paris, France
}

Perceptual decision-making is the subject of many experimental and theoretical studies. Most modeling analyses are based on statistical processes of accumulation of evidence. In contrast, very few works confront attractor network models' predictions with empirical data from continuous sequences of trials. Recently however, numerical simulations of a biophysical competitive attractor network model have shown that such a network can describe sequences of decision trials and reproduce repetition biases observed in perceptual decision experiments. Here we get more insights into such effects by considering an extension of the reduced attractor network model of Wong and Wang (2006), taking into account an inhibitory current delivered to the network once a decision has been made. We make explicit the conditions on this inhibitory input for which the network can perform a succession of trials, without being either trapped in the first reached attractor, or losing all memory of the past dynamics. We study in detail how, during a sequence of decision trials, reaction times and performance depend on nonlinear dynamics of the network, and we confront the model behavior with empirical findings on sequential effects. Here we show that, quite remarkably, the network exhibits, qualitatively and with the correct order of magnitude, post-error slowing and post-error improvement in accuracy, two subtle effects reported in behavioral experiments in the absence of any feedback about the correctness of the decision. Our work thus provides evidence that such effects result from intrinsic properties of the nonlinear neural dynamics.

Key words: attractor networks; perceptual decision-making; post-error adjustments; post-error slowing; reaction times

\section{Significance Statement}

Much experimental and theoretical work is being devoted to the understanding of the neural correlates of perceptual decisionmaking. In a typical behavioral experiment, animals or humans perform a continuous series of binary discrimination tasks. To model such experiments, we consider a biophysical decision-making attractor neural network, taking into account an inhibitory current delivered to the network once a decision is made. Here we provide evidence that the same intrinsic properties of the nonlinear network dynamics underpins various sequential effects reported in experiments. Quite remarkably, in the absence of feedback on the correctness of the decisions, the network exhibits post-error slowing (longer reaction times after error trials) and post-error improvement in accuracy (smaller error rates after error trials).

\section{Introduction}

Typical experiments on perceptual decision-making consist of series of successive trials separated by a short time interval, in which performance in identification and reaction times are mea-

\footnotetext{
Received April 20, 2018; revised 0ct. 30, 2018; accepted Nov. 18, 2018.

Author contributions: K.B. wrote the first draft of the paper; K.B. and J.-P.N. edited the paper; K.B. and J.-P.N. designed research; K.B. performed research; K.B. and J.-P.N. analyzed data; K.B. and J.-P.N. wrote the paper.

K.B. acknowledges a fellowship from the ENS Paris-Saclay. We thank Jerôme Sackur, Jean-Rémy Martin, and Laurent Bonnasse-Gahot for useful discussions, and the anonymous reviewers for helpful and constructive comments.

The authors declare no competing financial interests.

Correspondence should be addressed to Kevin Berlemont at kevin.berlemont@lps.ens.fr.

https://doi.org/10.1523/JNEUROSCI.1015-18.2018

Copyright $\odot 2019$ the authors $\quad 0270-6474 / 19 / 390833-21 \$ 15.00 / 0$
}

sured. The most studied protocol is the one of two-alternative forced-choice (TAFC) task (Ratcliff, 1978; Laming, 1979a; Vickers, 1979; Townsend and Ashby, 1983; Busemeyer and Townsend, 1993; Shadlen and Newsome, 1996); Usher and McClelland, 2001; Ratcliff and Smith, 2004). Several studies have demonstrated strong serial dependence in perceptual decisions between temporally close stimuli (Fecteau and Munoz, 2003; Jentzsch and Dudschig, 2009; Danielmeier and Ullsperger, 2011). Such effects have been studied in the framework of statistical models of accumulation of evidence (Dutilh et al., 2012), the most common theoretical approach to perceptual decisionmaking (Ratcliff, 1978; Ashby, 1983; Bogacz et al., 2006; Shadlen et al., 2006; Ratcliff and McKoon, 2008) or with a more complex 
attractor network with additional memory units specifically implementing a biasing mechanism (Gao et al., 2009).

Wang (2002) proposed an alternative approach to the modeling of perceptual decision-making based on a biophysical cortical network model of leaky integrate-and-fire neurons. The model is shown to account for random-dot experimental results from Shadlen and Newsome (2001) and Roitman and Shadlen (2002). This decision-making attractor network was first studied in the context of a task requiring to keep in memory the last decision. This working memory effect is precisely achieved by having the network activity trapped into an attractor state. However, in the context of consecutive trials, the neural activities have to be reset in a low activity state before the onset of the next stimulus. Bonaiuto et al. (2016) have considered a parameter range of weaker excitation where the working memory phase cannot be maintained. The main result is that the performance of the network is biased toward the previous decision, an effect which decreases with the intertrial time. Because of the slow relaxation dynamics in the model, we only study intertrial times $>1.5 \mathrm{~s}$. However, sequential effects have been reported for shorter intertrial times, such as 500 ms by Laming (1979a) and Danielmeier and Ullsperger (2011). Instead of decreasing the recurrent excitation, an alternative is to introduce an additional inhibitory input following a decision (Lo and Wang, 2006; Engel et al., 2015; Bliss and D'Esposito, 2017). Lo and Wang (2006) have proposed such a mechanism to account for the control of the decision threshold.

The purpose of the present paper is to revisit this issue of dealing with sequences of successive trials within the framework of attractor networks with a focus on intertrial times as short as $500 \mathrm{~ms}$. We do so by taking advantage of the reduced model of Wong and Wang (2006) which is amenable to mathematical analysis. This model consists of a network of two units, representing the pool activities of two populations of cells, each one being specific to one of the two stimulus categories. Wong and Wang (2006) derive the equations of the reduced model and choose the parameter values to preserve as much as possible the dynamical and behavioral properties of the original model. In line with Lo and Wang (2006), we take into account an inhibitory current originating from the basal ganglia, occurring once a decision has been made. We explore how the network nonlinear dynamics leads to serial dependence effects in TAFC tasks, and compare with empirical findings such as sequential bias in decisions (Cho et al., 2002) or post-error adjustments (Danielmeier and Ullsperger, 2011; Danielmeier et al., 2011). Our main finding is that the model reproduces two main post-error adjustments observed in the absence of feedback on the correctness of the decision: post-error slowing (PES) and post-error improvement in accuracy (PIA), with PES consisting of longer reaction times, and PIA of smaller error rates, for trials following a trial with an incorrect decision. We thus provide evidence that such effects result from nonlinearities in the neural dynamics.

\section{Materials and Methods}

We are interested in modeling experiments where a subject has to decide whether a stimulus belongs to one or the other of two categories, hereafter denoted $L$ and $R$. A particular example is the one of random-dot experiments (Shadlen and Newsome, 2001; Roitman and Shadlen, 2002), where a monkey performs a motion discrimination task in which it has to decide whether a motion direction, embedded into a random-dot motion, is toward left $(L)$ or right $(R)$. The general case is the one of categorical perception experiments, in which one can control the degree of ambiguity of the stimuli; e.g., psycholinguistic experiments with stimuli interpolating between two phonemes (Liberman et al., 1957), visual categorization experiments with continuous morphs from cats to dogs
(Freedman et al., 2003), etc. We focus on TAFC protocols in which no feedback is given on the correctness of the decisions.

We consider a decision-making recurrent network of spiking neurons governed by local excitation and feedback inhibition, as introduced and studied by Compte et al. (2000) and Wang (2002). Because mathematical analysis is harder to be performed for such complex networks, without a high level of abstraction (Miller and Katz, 2013), one must rely on simulations which, themselves, can be computationally heavy. For our analysis, we make use of the reduced firing-rate model of Wong and Wang (2006) obtained by a systematic reduction of the detailed biophysical attractor network model. The reduction aimed at faithfully reproducing not only the behavioral behavior of the full model, but also neural firing rate dynamics and the output synaptic gating variables. This is done within a mean-field approach, with calibrated simplified $F / I$ curves for the neural units, and in the limit of slow NMDA gating variables motivated by neurophysiological data. The full details were given by Wong and Wang (2006; their main text and supplemental Information).

Because this model has been built to reproduce as faithfully as possible the neural activity of the full spiking neural network, it can be used as a proxy for simulating the full spiking network (Engel and Wang, 2011; Deco et al., 2013; Engel et al., 2015). Here, we mainly make use of this model to gain better insights into the understanding of the model behavior. In particular, one can conveniently represent the network dynamics in a $2 \mathrm{~d}$ phase plane and rigorously analyze the network dynamics (Wong and Wang, 2006).

\section{A reduced recurrent network model for decision-making}

We first present the architecture without the corollary discharge (Wong and Wang (2006); Fig. 1A), which consists of two competing units, each one representing an excitatory neuronal pool, selective to one of the two categories, $L$ or $R$. The two units inhibit one another, while they are subject to self-excitation. The dynamics is described by a set of coupled equations for the synaptic activities $S_{L}$ and $S_{R}$ of the two units $L$ and $R$ :

$$
i \in\{L, R\}, \frac{\mathrm{d} S_{i}}{\mathrm{~d} t}=-\frac{S_{i}}{\tau_{S}}+\left(1-S_{i}\right) \gamma f\left(I_{i, t o t}\right) .
$$

The synaptic drive $S_{i}$ for pool $i \in\{L, R\}$ corresponds to the fraction of activated NMDA conductance, and $I_{i, \text { tot }}$ is the total synaptic input current to unit $i$. The function $f$ is the effective single-cell input/output relation (Abbott and Chance, 2005), giving the firing rate as a function of the input current:

$$
f\left(I_{i, t o t}\right)=\frac{a I_{i, t o t}-b}{1-\exp \left[-d\left(a I_{i, t o t}-b\right)\right]}
$$

where $a, b, d$ are parameters whose values are obtained through numerical fit.

The total synaptic input currents, taking into account the inhibition between populations, the self-excitation, the background current and the stimulus-selective current can be written as follows:

$$
\begin{aligned}
& I_{L, t o t}=J_{L, L} S_{L}-J_{L, R} S_{R}+I_{\text {stim }, L}+I_{\text {noise }, L}, \\
& I_{R, \text { tot }}=J_{R, R} S_{R}-J_{R, L} S_{L}+I_{\text {stim }, R}+I_{\text {noise }, R},
\end{aligned}
$$

with $J_{i, j}$ the synaptic couplings ( $i$ and $j$ being $L$ or $R$ ). The minus signs in the equations make explicit the fact that the inter-unit connections are inhibitory (the synaptic parameters $J_{i, j}$ being thus positive or null). The term $I_{\text {stim }, i}$ is the stimulus-selective external input. If $\mu_{0}$ denotes the strength of the signal, the form of this stimulus-selective current is as follows:

$$
\begin{aligned}
& I_{\text {stim }, L}=J_{A, e x t} \mu_{0}(1 \pm c) \\
& I_{s t i m, R}=J_{A, e x t} \mu_{0}(1 \mp c)
\end{aligned} .
$$

The sign, \pm , is positive when the stimulus favors population $L$, negative in the other case. The quantity $c$, between 0 and 1 , gives the strength of the signal bias. It quantifies the coherence level of the stimulus. For example, in the random-dot motion framework, it corresponds to the fraction of dots contributing to the coherent motion. In the following, we will give 
A

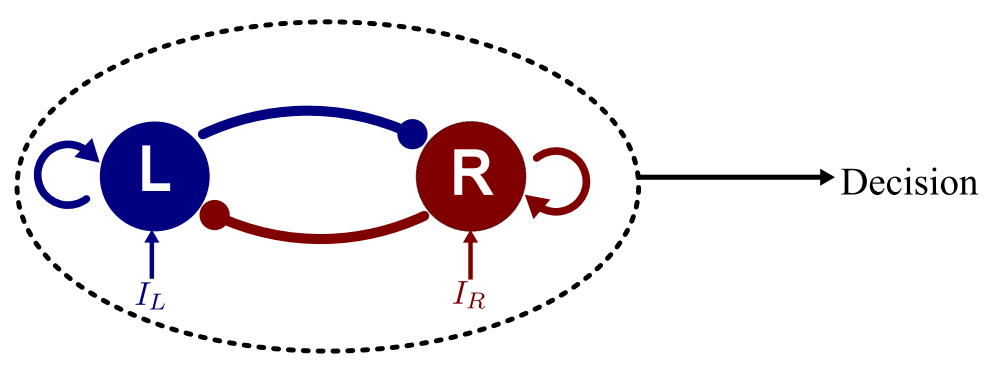

B

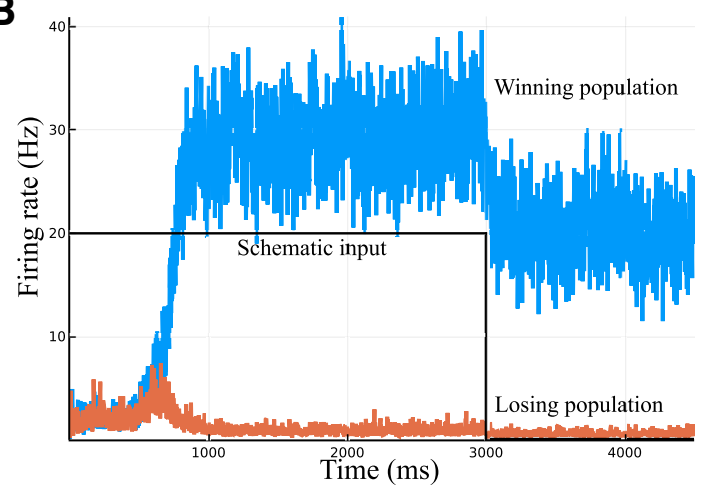

Figure 1. Two-variable model of Wong and Wang (2006). $\boldsymbol{A}$, Reduced two-variable model Wong and Wang (2006) constituted of two neural units, endowed with self-excitation and effective mutual inhibition. $\boldsymbol{B}$, Time course of the two neural activities during a decision-making task. At the beginning the two firing rates are indistinguishable. The firing rate that ramps upward (blue) represents the winning population, the orange one the losing population. A decision is made when one of the firing rate crosses the threshold of $20 \mathrm{~Hz}$. The black line represents the duration of the selective input corresponding to the duration of accumulation of evidence until the decision threshold is reached. This model shows working memory through the persistent activity in the network after the decision is made.

Table 1. Numerical values of the model parameters

\begin{tabular}{llll}
\hline Parameter & Value & Parameter & Value \\
\hline $\mathrm{a}$ & $270 \mathrm{~Hz} / \mathrm{nA}$ & $\sigma_{\text {noise }}$ & $0.02 \mathrm{nA}$ \\
$\mathrm{b}$ & $108 \mathrm{~Hz}$ & $\tau_{\text {noise }}$ & $2 \mathrm{mS}$ \\
$\mathrm{d}$ & $0.154 \mathrm{~s}$ & $I_{0}$ & $0.3255 \mathrm{nA}$ \\
$\gamma$ & 0.641 & $\mu_{0}$ & $30 \mathrm{~Hz}$ \\
$\tau_{S}$ & $100 \mathrm{~ms}$ & $J_{A, \text { ext }}$ & $5.2 \times 10^{-4} \mathrm{nA} / \mathrm{Hz}$ \\
$J_{L, L}=J_{R, R}$ & $0.2609 \mathrm{nA}$ & $J_{L, R}=J_{R, L}$ & $0.0497 \mathrm{nA}$ \\
$\theta$ & $20 \mathrm{~Hz}$ & & \\
$I_{C D, \max }$ & $0.035 \mathrm{nA}$ & $\tau_{\mathrm{CD}}$ & $200 \mathrm{~ms}$ \\
\hline
\end{tabular}

Top, Values as taken from Wong and Wang (2006). Bottom, Values of the additional parameters specific to the present model (see text).

this coherence level in percentage. Following Wang (2002), this input forms the pooling of the activities of middle temporal neurons firing according to their preferred directions. This input current is only present during the presentation of the stimulus and is shut down once the decision is made.

In the present model, in line with a large literature modeling decisionmaking, the input, Equation 5, is thus reduced to a signal parametrized by a scalar quantifying the coherence or degree of ambiguity of the stimulus. More global approaches consider the explicit coupling between the encoding and the decision neural populations, with a population of stimulus-specific cells for the coding layer (Beck et al., 2008; BonnasseGahot and Nadal, 2012; Engel et al., 2015). We believe that the main results presented here would not be affected by extending the model to take into account the coding stage, but we leave such study for further work.

In addition to the stimulus-selective part, each unit receives individually an extra noisy input, fluctuating around the mean effective external input $I_{0}$ :

$$
\tau_{\text {noise }} \frac{\mathrm{d} I_{\text {noise }, i}}{\mathrm{~d} t}=-\left(I_{\text {noise }, i}(t)-I_{0}\right)+\eta_{i}(t) \sqrt{\tau_{\text {noise }}} \sigma_{\text {noise }},
$$

with $\tau_{\text {noise }}$ a synaptic time constant that filters the (uncorrelated) white noises, $\eta_{i}(t), i=L, R$. For the simulations, unless otherwise stated, parameter values will be those shown in Table 1.

Initially the system is in a symmetric (or neutral) attractor state, with low firing rates and synaptic activities (Fig. $1 B$ ). On the presentation of the stimulus, the system evolves toward one of the two attractor states, corresponding to the decision state. In these attractors, the "winning" unit fires at a higher rate than the other. We are interested in reaction time experiments. In our simulations, we consider that the system has made a decision when for the first time the firing rate of one of the two units crosses a threshold $\theta$, fixed here at $20 \mathrm{~Hz}$. We have chosen this parameter value, slightly different from the one by Wong and Wang (2006), from the calibration of the extended model discussed below on sequential decision trials with short response-stimulus intervals (RSIs). We have checked that this does not affect the psychometric function of the network, the accuracy is unchanged and the reaction time is shifted by a constant.

\section{Extended reduced model: inhibitory corollary discharge}

Studies (Roitman and Shadlen, 2002; Ganguli et al., 2008) show that, during decision tasks, neurons' activity experiences a rapid decay following the responses (Roitman and Shadlen, 2002, their Figs. 7 and 9). Simulations of the above model show that even when the stimulus is withdrawn at the time of decision, the decrease in activity is not sufficiently strong to account for these empirical findings. Decreasing the recurrent excitatory weights does allow for a stronger decrease in activity, as shown by Bonaiuto et al. (2016). However, both the increase and the decay of activities are too slow, and the network cannot perform sequential decisions with RSIs $<1 \mathrm{~s}$. Hence the decrease in activity requires an inhibitory input at the time of the decision.

Such inhibitory mechanism has been proposed to originate from the superior colliculus (SC), controlling saccadic eye movements, and the basal ganglia-thalamic circuit, which plays a fundamental role in many cognitive functions including perceptual decision-making. Indeed, the burst neurons of the SC receive inputs from the parietal cortex and project to midbrain neurons responsible for the generation of saccadic eye movements (Scudder et al., 2002; Hall and Moschovakis, 2003). Thus the threshold crossing of the cortical neural activity is believed to be detected by the SC (Saito and Isa, 2003). In turn, the SC projects feedback connections on cortical neurons (Crapse and Sommer, 2009). At the time of a saccade, SC neurons emit a corollary discharge (CD) through these feedback connections (Sommer and Wurtz, 2008). The impact of this CD as an inhibition has been discussed in various contexts (Crapse and Sommer, 2008; Sommer and Wurtz, 2008; Yang et al., 2008). The generation of a corollary discharge resulting in an inhibitory input has been proposed and discussed in several modeling works, in the case of the modulation of the decision threshold in reaction time tasks (Lo and Wang, 2006), in the context of learning (Engel et al., 2015), and in a ring model of visual working memory (Bliss and D'Esposito, 2017).

We note here that, for simplicity and in accordance with the existing literature (Lo and Wang, 2006; Engel et al., 2015; Bliss and D'Esposito, 2017), we will be referring to the inhibitory current resulting from the corollary discharge as the corollary discharge.

In the context of attractor networks for decision tasks, Lo and Wang (2006) introduce an extension of the biophysical model of Wang (2002) that consists of modeling the coupling between the network, the basal ganglia, and the SC. The net effect is an inhibition onto the populations in charge of making the decision. Although Lo and Wang (2006) address the issue of the control of the decision threshold, they do not discuss the 
A

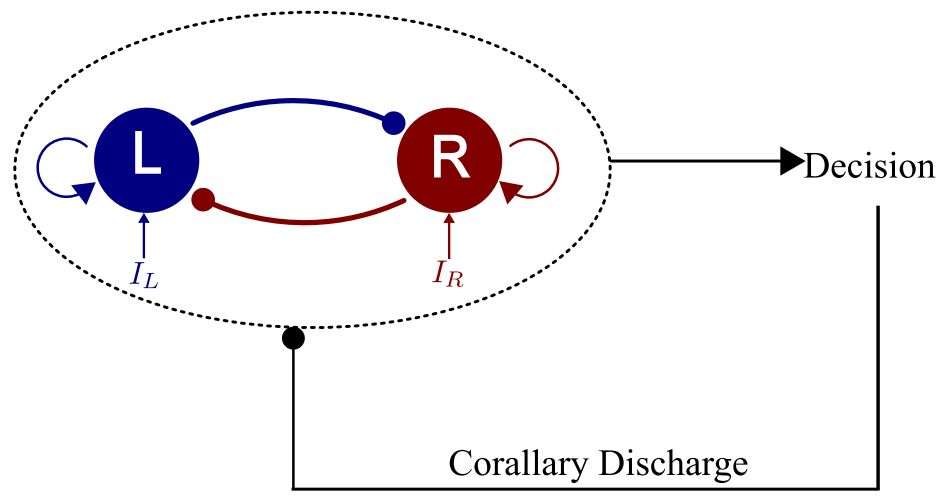

B

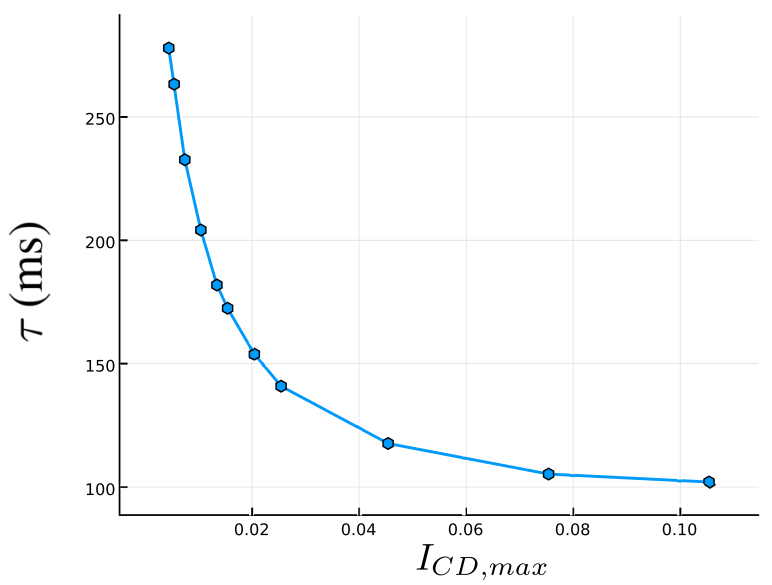

C

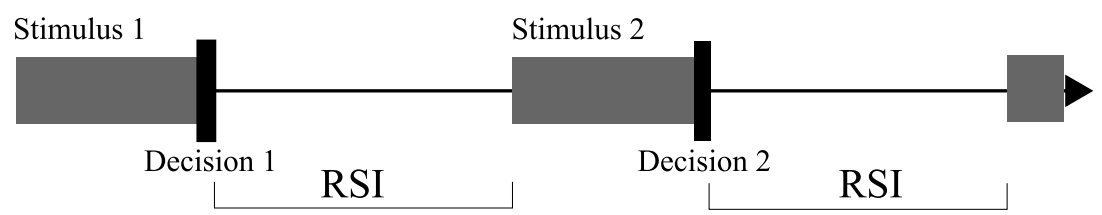

Figure 2. Extended version of the reduced model with the $C D$. $A$, The extension consists in adding the corollary discharge originating from the basal ganglia, an inhibitory input onto both units occurring just after a decision is made. $\boldsymbol{B}$, Relaxation time constant of the system during the RSI (that is the relaxation dynamics toward the neutral attractor), with respect to the corollary discharge amplitude. The values are obtained by computing the largest eigenvalue $\lambda$ of the dynamical system, Equations $1-6$, when presenting a constant $C D$. The time constant is given by the inverse of the eigenvalue, $\tau=-1 / \lambda$. C, The time-sketch of the simulations can be decomposed into a succession of identical blocks. Each block, corresponding to one trial, consists of: the presentation of a stimulus with a randomly chosen coherence (gray box), a decision immediately followed by the removal of the stimulus, a waiting time of constant duration corresponding to the RSI.

relaxation dynamics induced by the $\mathrm{CD}$, nor the effects on sequential decision tasks outside a learning context (Hsiao and Lo, 2013).

To analyze these effects with the reduced attractor network model, we assume that, after crossing the threshold, the network receives an inhibitory current, mimicking the joint effect of basal ganglia and SC on the two neural populations (Fig. $2 A$ ).

In the case of Engel et al. (2015), the function of the CD is to reset the neural activity to allow the network to learn during the next trial. For this, the form of the CD input is chosen as a constant inhibitory current for a duration of $300 \mathrm{~ms}$. However, such strong input leads to an abrupt reset to the neural state with no memory of the previous trial. We thus rather consider here a smooth version of this discharge, considering that the resulting inhibitory input has a standard exponential form (Finkel and Redman, 1983). The inhibitory input, $I_{\mathrm{CD}}(t)$, is then given by the following:

$$
I_{C D}(t)= \begin{cases}0 & \text { during stimulus presentation } \\ -I_{C D, \max } \exp \left(-\left(t-t_{D}\right) / \tau_{C D}\right) & \text { after the decision time, } \mathrm{t}_{\mathrm{D}}\end{cases}
$$

The relaxation time constant $\tau_{\mathrm{CD}}$ is chosen in the biological range of synaptic relaxation times and in accordance with the relaxation-time range of the network dynamics, $\tau_{\mathrm{CD}}=200 \mathrm{~ms}$ (Fig. $2 B$; see Discussion).

Therefore the input currents are modified as follows:

$$
\begin{aligned}
& I_{L, t o t}(t)=J_{L L} S_{L}(t)-J_{L, R} S_{R}(t)+I_{\text {stim }, L}(t)+I_{\text {noise }, L}(t)+I_{C D}(t), \\
& I_{R, t o t}(t)=J_{R R} S_{R}(t)-J_{R, L} S_{L}(t)+I_{\text {stim }, R}(t)+I_{\text {noise }, R}(t)+I_{C D}(t) .
\end{aligned}
$$

We can now study the dynamics of this system in a sequence of decision trials (protocol illustrated in Fig. 2C). We address here two issues: first, is there a parameter regime for which the network can engage in a series of trials; that is, for which the state of the dynamical system, at the end of the relaxation period (end of the RSI), is close to the neutral state (instead of being trapped in the attractor reached at the first trial); second, is there a domain within this parameter regime for which one expects to see se- quential effects (instead of a complete loss of the memory of the previous decision state).

In Figure 3 we illustrate the network dynamics between two consecutive stimuli during a sequence of trials, comparing the cases with and without the $\mathrm{CD}$. In the absence of the $\mathrm{CD}$ input, the network is not able to make a new decision different from the previous one (Fig. 3A). Even when the opposite stimulus is presented, the system cannot leave the attractor previously reached, unless in the presence of an unrealistic strong input bias. If however the strength $I_{\mathrm{CD} \text {, max }}$ is strong enough, the $\mathrm{CD}$ makes the system to escape from the previous attractor and to relax toward near the neutral resting state with low firing rates. If too strong, or in case of a too long RSI, at the onset of the next stimulus the neutral state has been reached and memory of past trials is lost. For an intermediate range of parameters, at the onset of the next stimulus the system has escaped from the attractor but is still on a trajectory dependent on the previous trial (Fig. 3B).

We have computed the time constant $\tau$ of the network during relaxation (during the RSI), with respect to the $\mathrm{CD}$ amplitude, $I_{\mathrm{CD} \text {, max }}$ (Fig. $2 B$ ). This computation is done for a $\mathrm{CD}$ with a constant amplitude, $I_{\mathrm{CD}}(t)=I_{\mathrm{CD} \text {, max }}$. One sees that, for $I_{\mathrm{CD} \text {, max }}$ of order $0.03 \sim 0.04 \mathrm{nA}$, the network time constant $\tau$ is four to five times smaller than the duration of the RSI. We choose the relaxation constant $\tau_{\mathrm{CD}}$ of the CD of the same order of magnitude (as in the above simulation where $\tau_{\mathrm{CD}}=200 \mathrm{~ms}$ ). With such value, at the onset of the next stimulus, the network state will still be far enough from the symmetric attractor, so that we can expect to observe sequential effects, as confirmed by the analysis in Results.

With the inhibitory CD, after the threshold is crossed by one of the two neural populations, there is a big drop in the neuronal activity (Fig. 3B), corresponding to the exit from the previous attractor state. This type of time course is in agreement with the experimental findings of Roitman and Shadlen (2002) and Ganguli et al. (2008), who measure the activity of LIP neurons during a decision task. They show that neurons that accumulate evidence during decision tasks experience rapid decay, or inhibitory suppression, of activity following responses, similar to Figure $3 B$ (but see Lo and Wang, 2006 for a related modeling study with spiking 
A
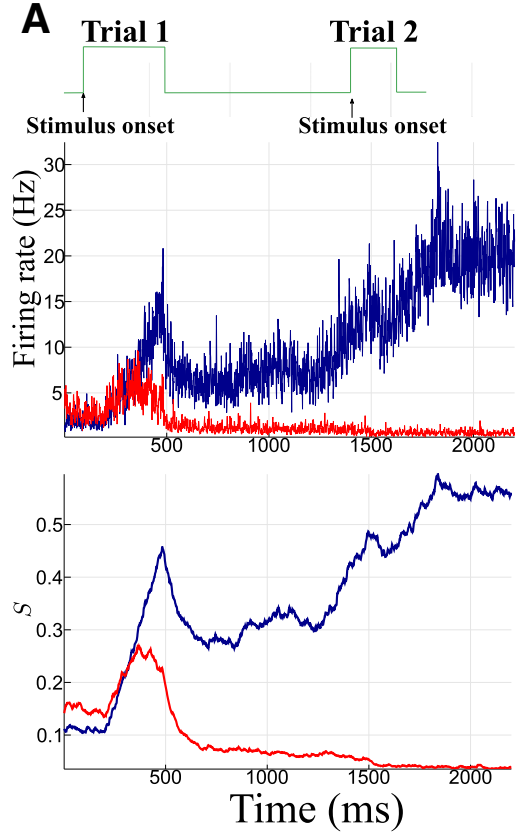
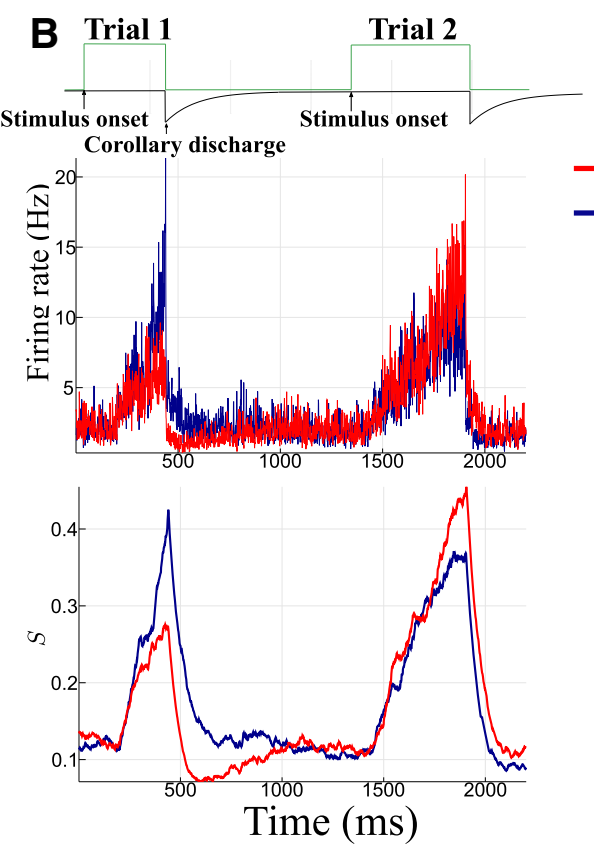

Figure 3. Time course of activities during two consecutive trials. $A$, Without CD. Top (green plot), Time course of the stimulations. The first stimulus belongs to category $L$, the second to category R. Middle, firing rates of the $L$ (blue) and $R($ red) neural pools. Bottom, corresponding synaptic activities. The neural activity becomes stuck in the attractor corresponding to the first decision. $\boldsymbol{B}$, With $C D$, with $I_{C D, \max }=0.035 \mathrm{nA}$. Top, Time course of the stimulations (green plot; same protocol as for $A$ ), and time course of the inhibitory current (black curve, represented inverted for clarity of the presentation). $\boldsymbol{B}$, Middle and Bottom, Neural and synaptic activities, respectively (L pool, blue; R pool, red). In that case, one observes the decay of activity after a decision has been made, and the winning population is different for the two trials.

neurons, or Gao et al., 2009 for rapid decay of neural activity with another type of attractor network).

We now derive the conditions on $I_{\mathrm{CD}}$ under which the network is able to make a sequence of trials. To this end, we analyze the dynamics after a decision has been made, during the RSI (hence during the period with no external excitatory inputs). The results are illustrated in Figure 4 on which we represent a sketch of the phase plane dynamics and a bifurcation diagram.

Consider first what would happen under a scenario of a constant, time independent, inhibitory input during all the RSI (Fig. 4A-D; formally, this corresponded to setting $\tau_{\mathrm{CD}}=+\infty$ in Eq. 7). At small values of the inhibitory current, the attractor landscape is qualitatively the same as in the absence of inhibitory current: in the absence of noise there is three fixed points, one associated with each category and the neutral one (Fig. $3 B$ ). At some critical value, of $\sim 0.0215 \mathrm{nA}$, there is a bifurcation (Fig. $4 D$ ); for larger values of the inhibitory current, only one fixed point remains, the neutral one (Fig. $4 D$ ). As a result, applying a constant CD would either have no effect on the attractor landscape, (current amplitude below the critical value), so that the dynamics remains within the basin of attraction of the attractor reaches at the previous trial; or would reset the activity at the neutral state (current amplitude above the critical value), losing all memory of the previous decision.

Now in the case of a CD with a value decreasing with time (Fig. $4 E-H$, scenario of an exponential decay), the network behavior will depend on where the dynamics lies at the time of the onset of the next stimulus. The dynamics, starting from a decision state (Fig. $4 F, G$, near the blue attractor), is more easily understood by considering the limit of slow relaxation (large time constant $\tau_{\mathrm{CD}}$ ). Between times $t$ and $t+\Delta t$, with $\Delta t$ small compared with $\tau$, the dynamics is similar to what it would be with a constant CD with amplitude $I_{\mathrm{CD}}(t)$. Hence if $I_{\mathrm{CD}}(t)$ is larger than the critical value discussed above, the dynamics "sees" a unique attractor, the neutral state, and is driven toward it. When $I_{\mathrm{CD}}(t)$ becomes smaller than the critical value, the system sees again three attractors, and finds itself within the basin of attraction of either the initial fixed point (corresponding to the previous decision; Fig. $4 F$ ), or of the neutral fixed point (Fig. $4 G)$. In the latter case, the network is able to engage in a new decision task.

To conclude, to have the network performing sequential decision tasks, one needs $I_{\mathrm{CD} \text {, max }}$ to be larger than the critical value $\left(\sim I_{\mathrm{CD}}=\right.$ $0.0215 \mathrm{nA}$; Fig. $4 H$ ), and, for a given value of $I_{\mathrm{CD} \text {, max }}$, to have a time constant $\tau_{\mathrm{CD}}$ large enough compared with the RSI for the system to relax close enough to the neutral attractor at the onset of the next stimulus. However, sequential effects may exist only if the current decreases sufficiently rapidly, so that the trajectory is still significantly dependent on the state at the previous decision. This justifies the choice of exponential decrease of the inhibitory current (Eq. 7) and the numerical value of $\tau_{\mathrm{CD}}=200 \mathrm{~ms}$. We note that recording from relay neurons, Sommer and Wurtz (2002) show that the signal corresponding to the CD last several hundreds of milliseconds. This time scale falls precisely in the range of values of the relaxation time constant of the model (Fig. $2 B$ ), and corresponds to values for which, as we will see, the model shows sequential effects.

\section{Numerical simulations design and statistical tests}

Numerical simulations. The simulation of sequential decision-making is as follows: a stimulus with a randomly chosen coherence is presented until the network reaches a decision (decision threshold crossed). The decision is immediately followed by the removal of the stimulus, and a relaxation period during the RSI. Then a new stimulus is presented, initiating the next trial (Fig. 2C). The set of dynamical equations (Eqs. 1, 6), with the definitions (Eqs. 2, 5, 7-9), is numerically integrated using Euler-Maruyama method with a time step of $0.5 \mathrm{~ms}$. At the beginning of a simulation, the system is set in a symmetric state $S_{L}=S_{R}=s_{0}$, with low firing rates and synaptic activities, $s_{0}=0.1$. We compute the instantaneous population firing rates, or the synaptic dynamical variables $S_{L}$ and $S_{R}$, by averaging on a time window of $2 \mathrm{~ms}$, slided with a time step of $1 \mathrm{~ms}$. The accuracy of the network's performance is defined as the percentage of trials for which the units crossing the threshold corresponds to the stronger input. For data analysis we mainly work with the variables $S_{L}$ and $S_{R}$, which are analog to the firing rates $R_{L}$ and $R_{R}$ [because they are monotonic functions of $S_{L}$ and $S_{R}$ (Wong and Wang, 2006) but are less noisy; Fig. 3]. We consider that the system has made a decision when for the first time the firing rate of one unit crosses a threshold $\theta$, fixed at 20 $\mathrm{Hz}$. The reaction time during one trial is defined as the time needed for the network to reach the threshold from the onset of the input stimulus. 
A

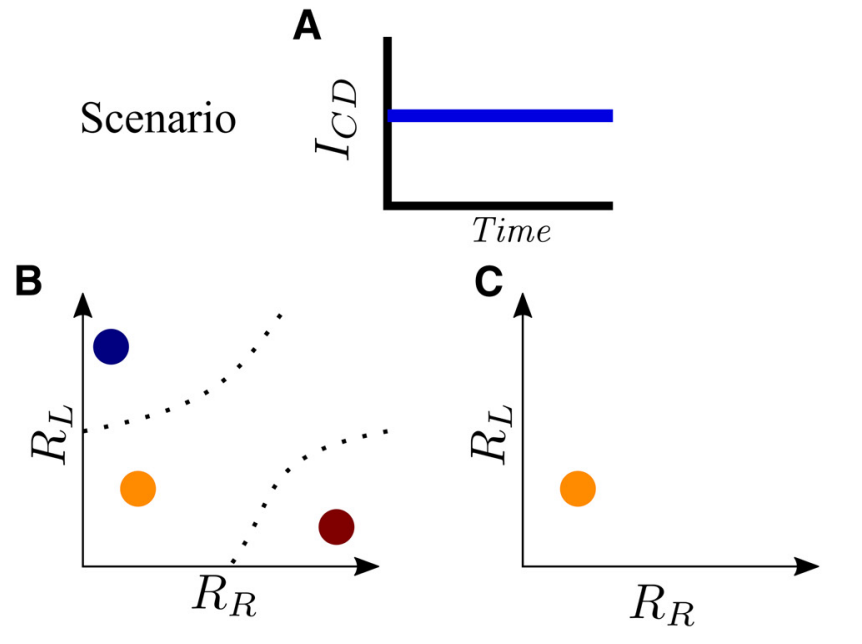

D

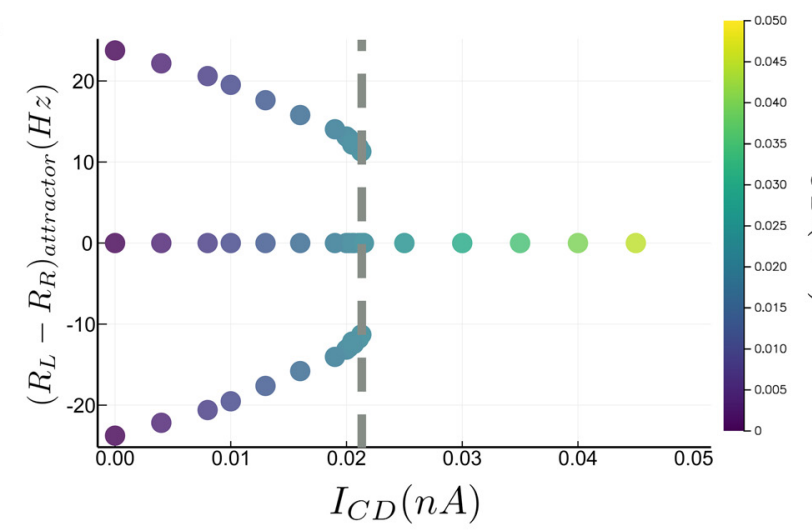

Neutral attractor
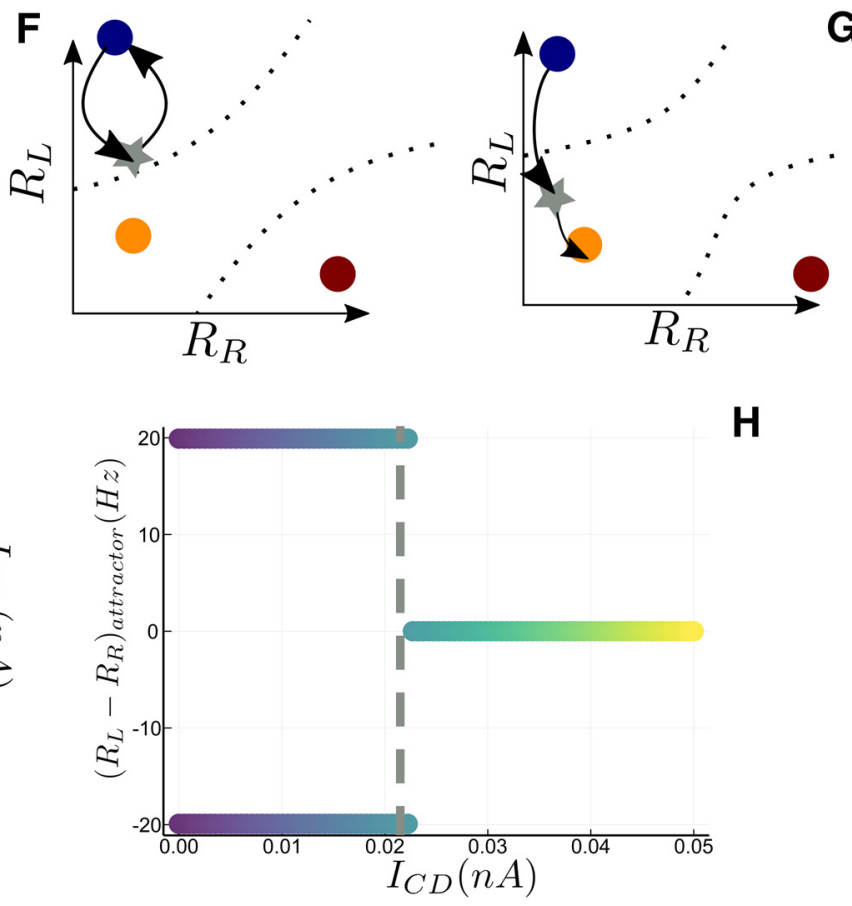

Attractor R
E

\section{Scenario}

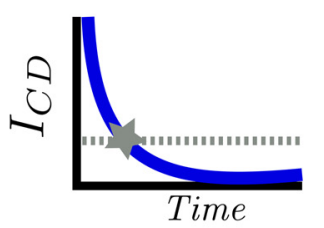

G

Figure 4. Bifurcation diagram of sequential decision-making, for two scenario of $I_{C D}$. $\boldsymbol{A}$, Scenario with a constant value of the inhibitory current for $\boldsymbol{B}-\boldsymbol{D}$. $\boldsymbol{B}$, Phase plane representation of the attractors at low $I_{C D}$ (below the critical value). $C$, Phase plane representation of the attractor landscape at high $I_{C D}$ (above the critical value). Only the neutral attractor exists, corresponding to $D$ (right). $D$, Attractors state (as the difference in firing rates, $R_{L}-R_{R}$ ) with respect to $I_{C D}$. The gray line, at $I_{C D}=0.0215 \mathrm{nA}$, represents the bifurcation point. On the left side three attractors exists, on the right side only the neutral one exists. The case without inhibitory current corresponds to $I_{C D}=0 \mathrm{nA}$. $\boldsymbol{E}$, Scenario with an inhibitory current decreasing exponentially with time, for $\boldsymbol{F}-\boldsymbol{H}$. The dashed line corresponds to $I_{C D}=0.0215 \mathrm{nA}$, value at which the bifurcation at constant $I_{C D}$ occurs $(\boldsymbol{D})$. The time at which the current amplitude crosses this value is denoted by the gray star in $\boldsymbol{E}$ and $\boldsymbol{F}$. $\boldsymbol{F}, \boldsymbol{S}$ chematic phase-plan dynamics corresponding to the left side of $\boldsymbol{H}$. The blue attractor corresponds to the starting point and the black arrow represents the dynamics. At the time $I_{C D}$ becomes $I_{O w}$. $\mathrm{nA}$ (gray star), the system is still within the basin of attraction of the initial attractor. Hence, it goes back to the initial attractor. $\mathbf{G}$, Schematic phase-plan dynamics corresponding to the $\boldsymbol{H}$ (right). At the time $I_{C D}$ becomes lower than $0.0215 \mathrm{nA}$, the system lies within the basin of attraction of the neutral attractor. Hence, the dynamics continues toward the neutral attractor. Those conditions are the ones needed for sequential decision-making. $\boldsymbol{H}$, Attractors that can be reached when starting from a decision state, for the relaxation dynamics under the scenario represented on $\boldsymbol{E}$. On the left side of the dashed gray line, the value of $I_{C D, \max }$ is too weak and the network remains locked to the attractor corresponding to the previous decision state. On the right side the network dynamics lies within the basin of attraction of the neutral attractor, allowing the network to engage in a new decision task.

We neglect the possible additional time because of motor reaction and signal transduction. In addition to the reaction times, we compute the discrimination threshold, which is linked to the accuracy. The definition is based on the use of a Weibull function commonly used to fit the psychometric curves (Quick, 1974). That is, one writes the performance (mean success rate) as follows:

$$
\operatorname{Perf}(c)=1-0.5 \exp \left(-(c / \alpha)^{\beta}\right),
$$

where $\alpha$ and $\beta$ are parameters. Then, for $c=\alpha, \operatorname{Perf}(\mathrm{c})=1-0.5$ $\exp (-1) \sim 0.82$.

Hence one defines the discrimination threshold as the coherence level at which the subject responds correctly $82 \%$ of the time.

We list in Table 1 the model parameters that correspond to the one of the simulations. For Figures 5 and 7 we have used continuous sequences of 1000 trials averaged over 24 independent simulations, allowing to more specifically compare with the experiments of Bonaiuto et al. (2016) done with 24 subjects. Figures 9 to 16 present results obtained for sequences of 1000 trials averaged over 50 independent simulations to allow for a better statistical analysis. The number of sequences, 1000, is a typical order of magnitude in experiments (Bonaiuto et al. 2016; Danielmeier and Ullsperger, 2011).

Statistical tests. Following Benjamin et al. (2018), we consider a $p$ value of 0.005 as a criterion for rejecting the null hypothesis in a statistical test. To assess whether the distributions of two continuous variables are different, we make use of the Kolmogorov-Smirnov test (Hollander et al., 2014), and in the case of discrete variable distributions we use the Anderson-Darling test (Shorack and Wellner, 2009). For very large samples, we use the energy distance (Rizzo and Székely, 2016), which is a metric distance between the distributions of random vectors. We use the associated E-statistic (Szekely and Rizzo, 2013) for testing the null hypothesis that two random variables $X$ and $Y$ have the same cumulative distribution functions. For testing whether the means of two samples are different we make use of the unequal variance test (Welch's test; Hollander et al., 2014).

Software and code accessibility. For the simulations we made use of the Julia language (Bezanson et al., 2017). The code of the simulations can be obtained from the corresponding author upon request. We made use of the XPP software (Ermentrout and Mahajan, 2003) for the phase-space 
A

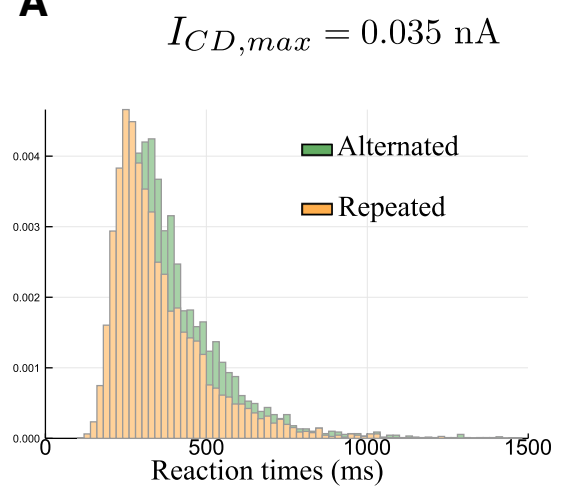

B

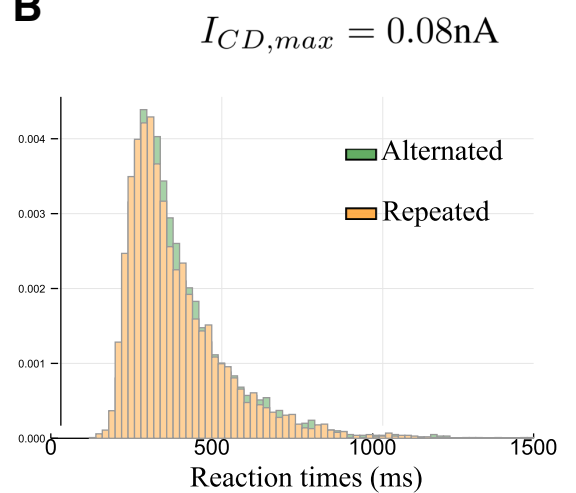

C

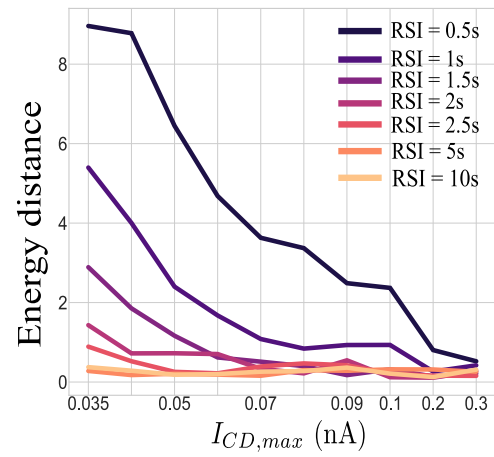

Figure 5. Histogram of the reaction times. Simulations run at $(\boldsymbol{A}) I_{\mathrm{CD} \text {,max }}=0.035 \mathrm{nA}$ and $(\boldsymbol{B}) I_{\mathrm{CD} \text {,max }}=0.08 \mathrm{nA}$, with a RSI of $1 \mathrm{~s}$. The green histogram corresponds to the Alternated case, that is when the decisions made at the $n$th and $n$th +1 trials are different. The orange histogram corresponds to the Repeated case, that is when the decisions made at the $n$th and $n$th +1 trials are identical. C, Energy distance between the repeated and alternated histograms. The $x$-axis represents the strength of the corollary discharge, and the color codes the duration of the RSI in seconds.

analysis and the computation of the relaxation time constant of the dynamical system. Figures 9, 10, 11, 12, and 19 were realized using Python and the other are in the same language as the simulations. The E-statistics tests were performed using the R-Package: energy package (Rizzo and Székely, 2014).

\section{Results}

\section{Sequential dynamics and choice repetition biases}

The dynamical properties described above give that, for the appropriate parameter regime, the RSI relaxation leads to a state which is between the previous decision state and the neutral attractor. If it is still within the basin of attraction of the previous decision state at the onset of the next stimulus, one expects sequential biases. This mechanism is similar to the one discussed by Bonaiuto et al. (2016). However, the relaxation mechanisms are different, as discussed in the Introduction. This results in different quantitative properties, notably and quite importantly in the time scale of the relaxation, which is here more in agreement with experimental findings (Cho et al., 2002).

We will specifically show that nonlinear dynamical effects are at the core of post-error adjustments. As a preliminary step, it is necessary to investigate the occurrence of sequential effects in our model. We do so by describing more precisely the intertrial dynamics: we need to specify where the network state lies at the onset of a new stimulus, with respect to the boundaries between the basins of attraction. We take advantage of this analysis to explore response repetition bias as studied by Bonaiuto et al. (2016), and to confront the model behavior with other empirical findings (Laming, 1979a; Cho et al., 2002). In all the following, we study the model properties in function of the two parameters, the amplitude of the corollary discharge, $I_{\mathrm{CD} \text {, max }}$, and the duration of the RSI.

\section{Network behavior: reaction times biases}

After running simulations of the network dynamics with the protocol of Figure $2 C$, we analyze the effects of response repetition by separating the trials into two groups, the Repeated and Alternated cases. The repeated (respectively alternated) trials are those for which the decision is identical to (respectively, different from) the decision at the previous trial. Note that we do not consider whether the stimulus category is repeated or alternated: the analysis is based on whether the decision is identical or different between two consecutive trials (Fleming et al., 2010; PadoaSchioppa, 2013). Such analysis is appropriate, because the effects under consideration depend on the levels of activity specific to the previous decision. We run a simulation of 1000 consecutive trials, each of them with a coherence value randomly chosen between 20 values in the range $(-0.512,0.512)$. We do so for two values of the $\mathrm{CD}$ amplitude, $I_{\mathrm{CD}, \max }=0.035 \mathrm{nA}$ and $I_{\mathrm{CD}, \max }=$ $0.08 \mathrm{nA}$, with a RSI of $1 \mathrm{~s}$, the other parameters being given on Table 1 .

We find that the distribution of coherence values are identical for the two groups, for both values of $I_{\mathrm{CD} \text {, max }}$ (Anderson-Darling test, $p=0.75$ and $p=0.84$, respectively). We study the reaction times separately for the two groups, and present the results in Figure 5. In Figure $5 C$ we represent the so called energy distance (Szekely and Rizzo, 2013; Rizzo and Székely, 2016) between the repeated and alternated reaction-time distribution. As we can observe, the distance decreases, hence the sequential effect diminishes, as the $\mathrm{CD}$ amplitude $I_{\mathrm{CD} \text {,max }}$ increases. For the specific case of Figures 5, $A$ and $B$, the corresponding E-statistic for testing equal distributions leads to the conclusion that in the case $I_{\mathrm{CD} \text {, max }}=$ $0.035 \mathrm{nA}$, the two reaction-time distributions are different ( $p=$ $0.0019)$. This implies that the behavior of the network is influenced by the previous trial. We observe a faster mean reaction time ( $\sim 55 \mathrm{~ms}$ ) when the choice is repeated (Fig. $5 A$ ), with identical shape of the reaction times distributions. The difference in means is of the same order as found by Cho et al. (2002) in experiments on 2AFC tasks. On the contrary, for $I_{\mathrm{CD} \text {, } \max }=0.08$ $\mathrm{nA}$ (Fig. $5 B$ ), the two histograms cannot be distinguished (Estatistic test, $p=0.25$ ).

We have checked that increasing the RSI has a similar effect to increasing the CD amplitude. We observe sequential effects for RSI values in the range $0.5-5 \mathrm{~s}$, in accordance with two-choice decision-making experiments, where such effects are observed for RSI < 5 s (Rabbitt and Rodgers, 1977; Laming, 1979a; Soetens et al., 1985).

\section{Neural correlates: dynamic analysis}

With the relaxation of the activities induced by the $\mathrm{CD}$, the state of the network at the onset of the next stimulus lies in-between the attractor state corresponding to the previous decision, and the neutral attractor state. When averaging separately over repeated and alternated trials, we find, as detailed below, that this relaxation dynamic has different behaviors depending on whether the next decision is identical or different from the pre- 
A
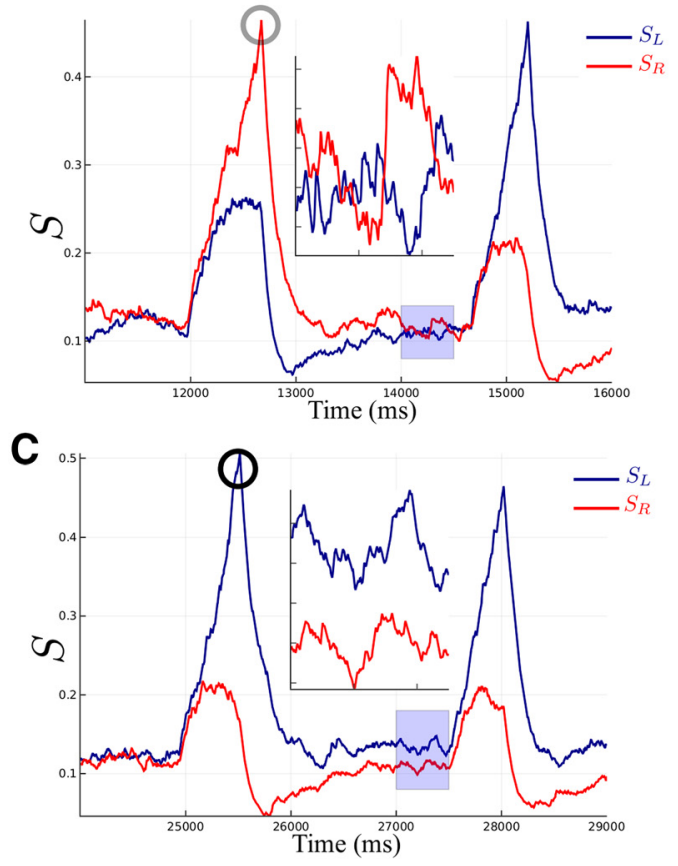
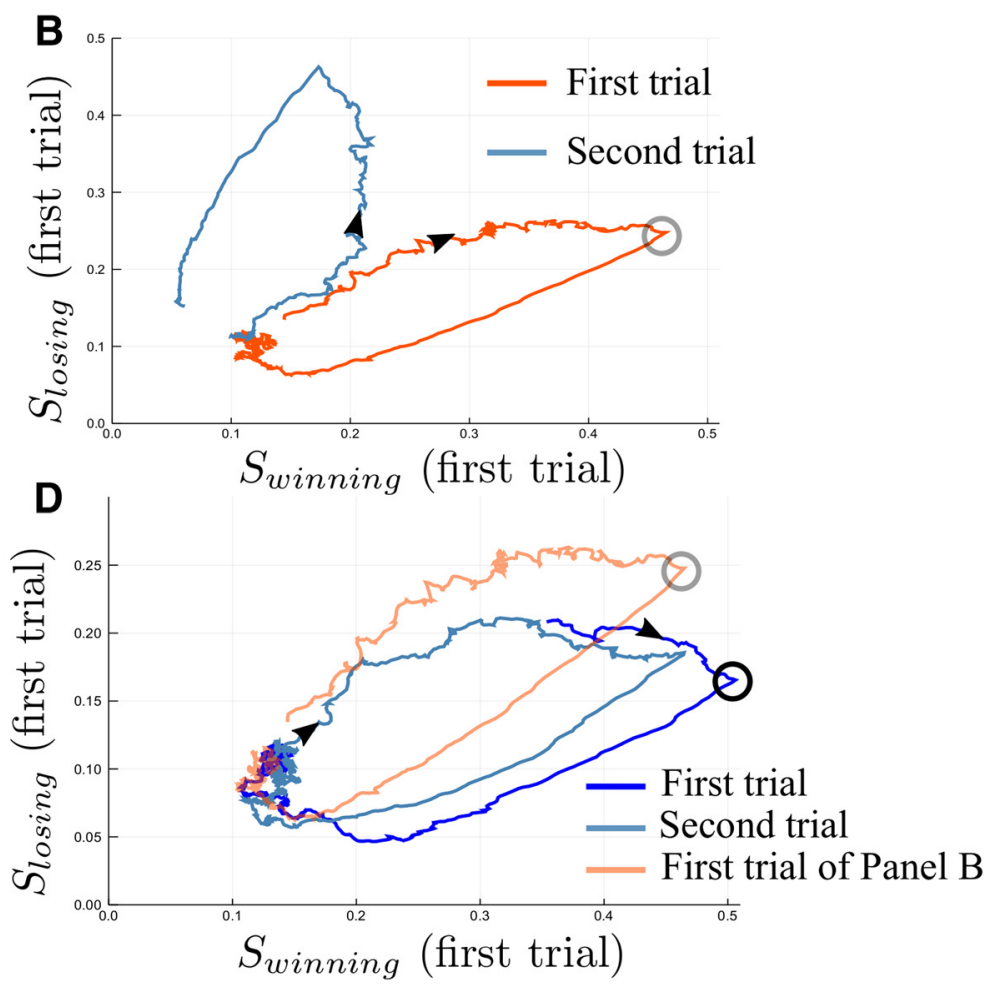

Figure 6. Network activity during two consecutive trials. $\boldsymbol{A}, \boldsymbol{B}$, The alternated case where the decision made is $R$ then $L$. $\boldsymbol{C}, \boldsymbol{D}$, The case where decision $L$ is made and repeated. $\boldsymbol{A}, \boldsymbol{C}$, The time course activities of the network. The light blue zone is zoomed to better see the dynamics just before the onset of the second stimulus. The red and blue curves correspond to the activities of, respectively, the $R$ and $L$ network units. $\boldsymbol{B}, \boldsymbol{D}$, Represent, respectively, the $\boldsymbol{A}$ and $\boldsymbol{C}$ dynamics in the phase-plane coordinates. $\boldsymbol{B}$, The dynamics evolve from dark red (first trial) to light blue (second trial), and ( $\boldsymbol{D}$ ) from dark blue (first trial) toward light blue (second trial). The gray, respectively black, circles identify the same specific point during the dynamics in $\boldsymbol{A}$ and $\boldsymbol{B}$, respectively, $\boldsymbol{C}$ and $\boldsymbol{D}$. The circles are not at the exact same value because the decision threshold is on the firing rates and not for the synaptic activities. To compare the alternated and repeated cases $\boldsymbol{A}$ and $\boldsymbol{B}$ and $\boldsymbol{C}$ and $\boldsymbol{D}$, the dark red curve of $\boldsymbol{B}$, is reproduced on $\boldsymbol{D}$ as a light orange curve.

vious one. Note that this is a statistical effect which can only be seen by averaging over a very large number of trials.

In Figure 6 we compare two examples of network activity, one with an alternated choice, and one with a repeated choice, by plotting the dynamics during two consecutive trials. We observe in Figure 6A, the alternated case, that previous to the onset of the second stimulus (light blue rectangle) the activities of the two populations are at very similar levels. In contrast, for the case of a repeated choice, Figure $6 C$, the activities are well separated, with higher firing rates.

In Figure $6 B$ we give a classical phase-plane representation of the network dynamics during two consecutive trials, with the axes as the synaptic activities of the winning versus loosing neuronal populations in the first trial. One sees a trajectory starting from the neutral state, going to the vicinity of the attractor corresponding to the first decision, and then relaxing to the vicinity of the neutral state (as illustrated in Fig. $4 G$ ). Then the trajectory goes toward the attractor corresponding to the next decision, different from the first one. This aspect of the dynamics is similar to what is obtained by Gao et al. (2009) with another type of attractor network. We show in Figure $6 D$ the phase-plane dynamics in the case of a repeated choice (trajectory in blue). On this same panel, for comparison we reproduce in light red the dynamics, shown in Figure $6 B$, during the first trial in the alternated case. As can be seen in Figure $6 D$, the network states at the time of decision are different depending on whether the network makes a decision identical to, or different from, the one made at the previous trial.

To check the statistical significance of these observations, we represent in Figure 7 the mean activities during the RSI, obtained by averaging the dynamics over all trials, separately for the alternated and repeated groups. As expected, for small values of $I_{\mathrm{CD} \text {,max }}(0.035 \mathrm{nA})$, the two dynamics are clearly different. This difference diminishes during relaxation. However at the onset of the next stimulus we can still observe some residues, statistically significant according to an Anderson-Darling test done on the $500 \mathrm{~ms}$ before the next stimulus (between winning population, $p=0.0034$, between losing population $p=3.2 \times 10^{-8}$ ).

Looking at Figure $7 A$, we observe that the ending points of the alternated and repeated relaxations are biased with respect to the symmetric state. At the beginning of the next stimulus the network is already in the basin of attraction of the repeated case. Hence, it will be harder to reach the alternated attractor stated (in the green region). When increasing $I_{\mathrm{CD} \text {, max }}$ (Fig. $7 B$ ), we observe that the ending state of the relaxation is closer to the attractor state. Hence, the biases in sequential effects disappear because at the beginning of the next stimuli the network starts from the symmetric (neutral) state. The same analysis holds for longer RSI, the dynamics are almost identical (Anderson-Darling test: between winning population, $p=0.25$, between losing population $p=0.4$ ), and both relaxation end near the neutral attractor state. The bias depending on the next stimulus is not observed anymore, and the sequential effect on reaction time hence disappears.

Note that the sequential effects only depend on whether or not the states at the end of the relaxation lie on the basin boundary. However, we have just seen that the effects can also be observed at the level of the relaxation dynamics, because the trajectories for alternated and repeated cases are identical when there is no effect, and different in the case of sequential effects. 
A

-Alternated

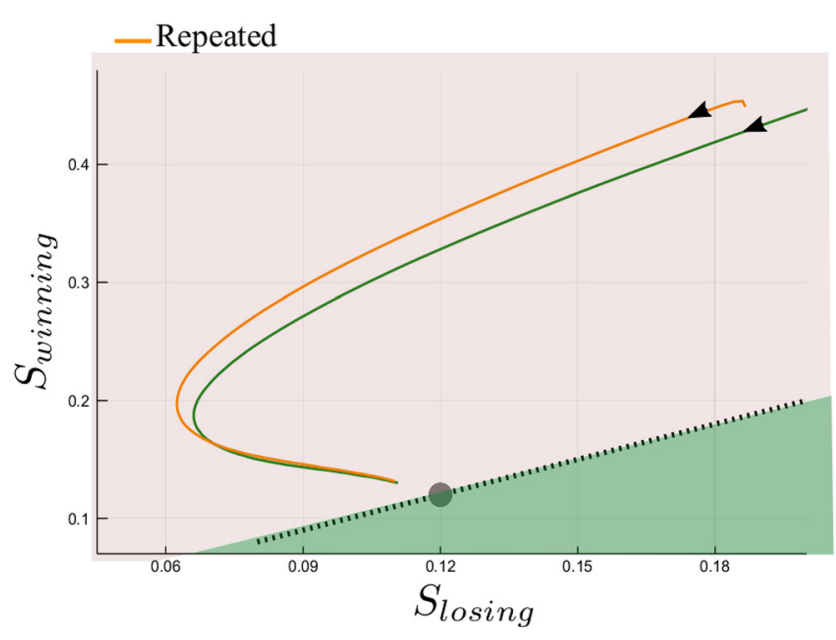

B

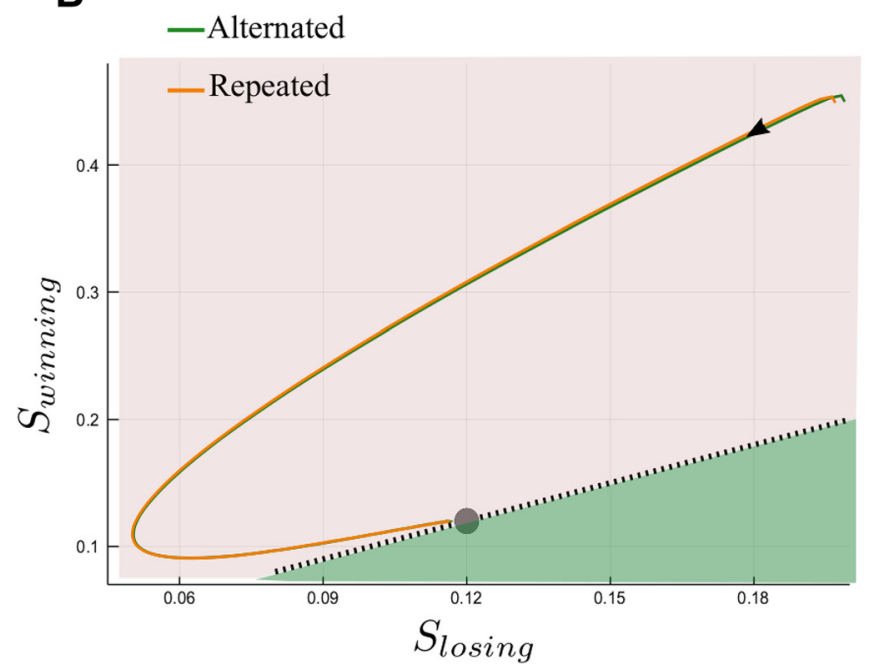

Figure 7. Phase plane dynamics. Dynamics of the decaying activity between two successive trials $(\boldsymbol{A})$ for $I_{C D \text {, } m a x}=0.035 \mathrm{nA}$, and $(\boldsymbol{B})$ for $I_{C D, \max }=0.08 \mathrm{nA}$. The synaptic activity is averaged over all trials separately for each one of the two groups: alternated (green) and repeated (orange). The axis are $S_{\text {winning }}$ and $S_{\text {losing }}$ (not $S_{R}$ and $S_{L}$ ) corresponding to the mean synaptic activity of, respectively, the winning and the losing populations for this trial. Note the difference in scale of the two axes. The time evolution along each curve follows the black arrow. The dashed black line denotes the symmetric states $\left(S_{L}=S_{R}\right)$ of the network, and the gray circle the neutral attractor. The shadow areas represent the basins of attraction (at low coherence levels) for the repeated and alternated trials, respectively, pink and green.
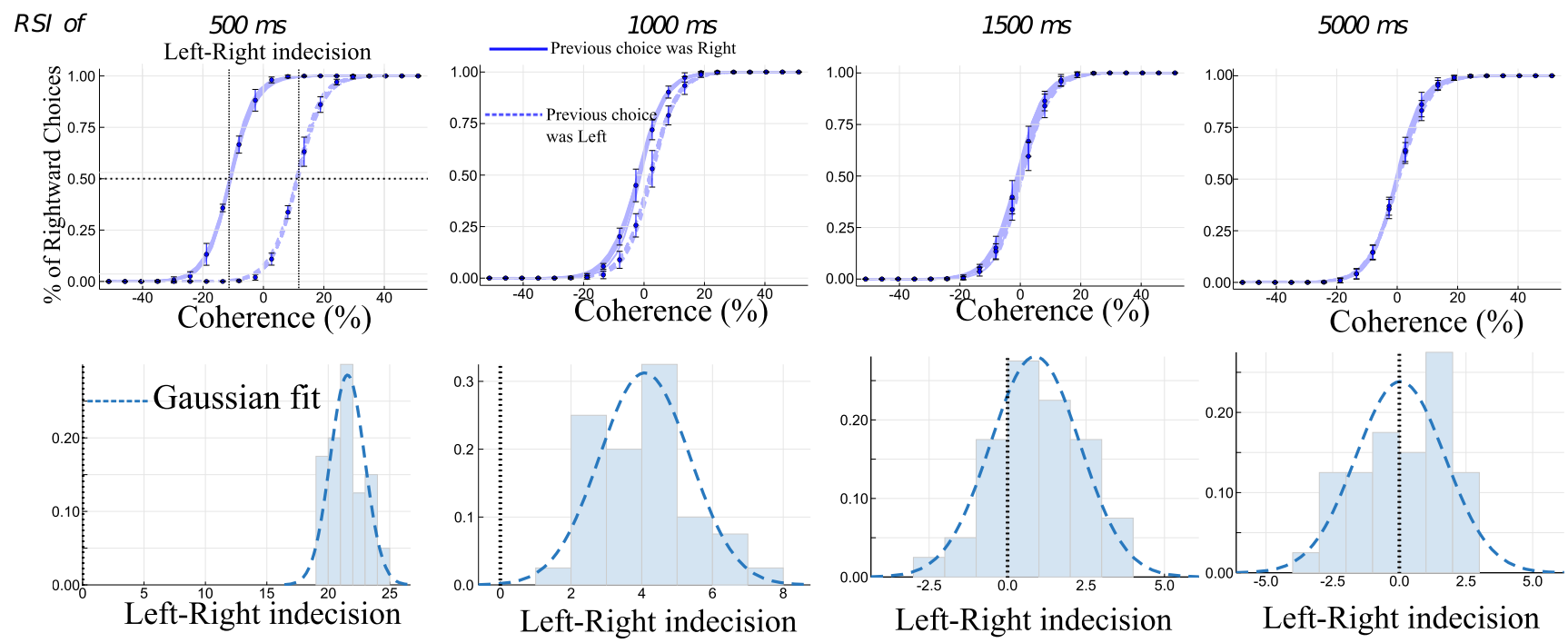

Figure 8. Repetition biases for several RSI values. Top, Percentage of Right choices, with respect to coherence level, depending on the previous choice (Left or Right). The blue points represents the mean accuracy (on 24 simulations) and the confidence interval at 95\% (bootstrap method). The blue lines denote the fit (of all simulations) by a logistic regression of all (plain, previous choice was Right; dashed, previous choice was Left). Bottom, Histogram of the Left-Right indecision point (on 24 simulations to stay in the experimental range). It characterizes the fact that the positive shift in the indecision point is increased for small RSI. The mean of the indecision point shift decreases with longer RSIs.

The analysis of the dynamics also leads to expectations for what concerns the bias in accuracy toward the previous decision. Indeed, this can be deduced from Figure 7. If the choice at the previous trial was $R$ (respectively $L$ ), then, at the end of the relaxation, the network lies closer to the basin of attraction of attractor $R$ (respectively $L$ ). Thus when presenting the next stimulus, the decision will be biased toward the previous state, so that the probability of making the same choice will be greater than the one of making the opposite choice. Otherwise stated, given the stimulus presented at the current trial, the probability to make the choice $R$ will be greater when the previous choice was also $R$, than when the previous choice was $L$. Numerical simulations confirm this analysis, as illustrated on Figure 8. The RSI dependency is statistically significant (generalized linear model: $r=-3.9, p<0.0001$ ). For small RSI (500 ms), the decision is biased toward the previous one, and for RSI of several seconds this effect disappears. These results are in agreement with experimental findings of Bonaiuto et al. (2016). The authors studied response repetition biases in human with RSIs of at least $1.5 \mathrm{~s}$. In these experiments, they measure the Left-Right indecision point, that is the level of coherence resulting in chance selection. Compared with the repeated case, they find that the indecision point for the alternated case is at a higher coherence level, and this shift decreases as the RSI increases.

Sequential decision effects have also been analyzed within the drift-diffusion model (DDM) framework (Farrell and Ludwig, 2008; Goldfarb et al., 2012). Behavioral data can be fitted by different choices of starting points, and possibly of thresholds 

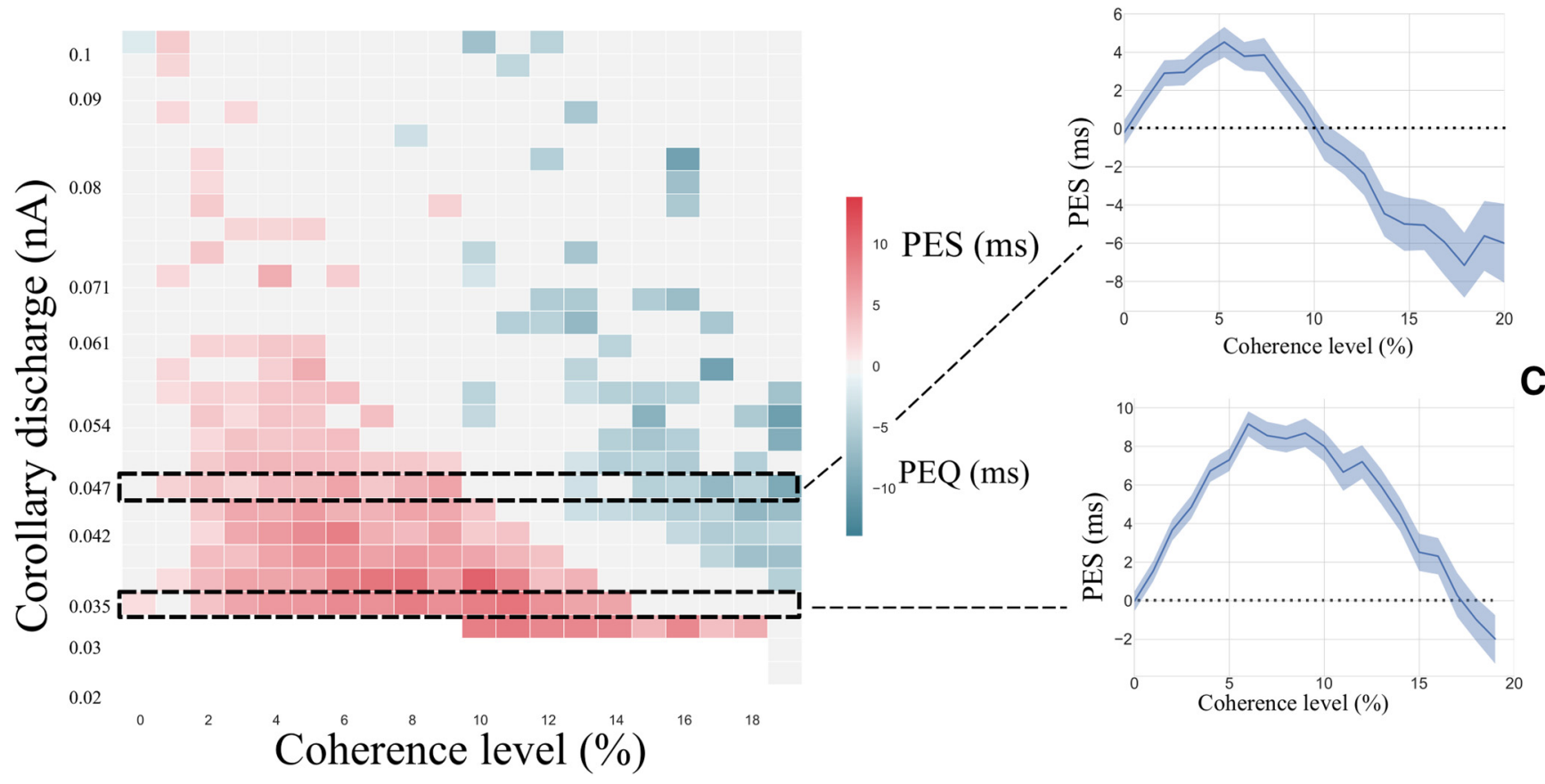

Figure 9. PES in the simulated network at a RSI of $500 \mathrm{~ms}$. A, Phase diagram of the PES effect at RSI of $500 \mathrm{~ms}$. The bottom white zone corresponds to parameters where sequential decision-making is impossible as the network is unable to leave the attractor state during the RSI. The red square corresponds to regions where PES is observed, and the blue ones where PEQ is observed (the darker the color, the stronger the effect). The black dashed squares correspond to specific regions where $\boldsymbol{B}$ and $\boldsymbol{C}$ zoom. $\boldsymbol{B}$, PES effect (ms) with respect to the coherence level at $I_{\mathrm{CD}, \max }=0.047 \mathrm{nA}$. The light blue zone corresponds to the bootstrapped (Efron and Tibshirani, 1994) confidence interval at 95\%. C, PES effect (ms) with respect to the coherence level at $I_{\mathrm{CD}, \max }=$ $0.035 \mathrm{nA}$. The light blue zone corresponds to the bootstrapped confidence interval at $95 \%$.

(Goldfarb et al., 2012). The modification of the starting point in a DDM framework is analog to the effect of the relaxation in our model. However, most works based on DDM make a post hoc analysis of empirical data, with separate fits for alternated and repeated cases.

To conclude this section, at the time of decision, the winning population has a firing rate higher than the losing population. After relaxation, at the onset of the next stimulus, the two neural pools have more similar activities, but are still sufficiently different, that is the dynamics is still significantly away from the neutral attractor. At the onset of the next stimulus, the systems finds itself in the basin of attraction of the attractor associated to the same decision as the previous one. This results in a dynamical bias in favor of the previous decision. The probability to make the same choice as the previous one is then larger than the one of the other choice, and the reaction time, for making the same choice (repeated case), is shorter than for making the opposite choice (alternated case). In accordance with these results, studies on the LIP, SC, and basal ganglia have found that the baseline activities before the onset of the stimuli can reflect the probabilities of making the saccade, under specific conditions (Lauwereyns et al., 2002; Ding and Hikosaka, 2006; Rao et al., 2012). Our model shows that these modulations of the baseline activities can be understood as resulting from the across-trial dynamics of the decision process.

\section{Post-error effects}

Post-error adjustments on reaction times

The most interesting and well established effect is the one of PES (Laming, 1979a; for review, see Danielmeier and Ullsperger, 2011). It consists of prolonged reaction times in trials following an error, compared with reaction times following a correct trial.
This effect has been observed in a variety of tasks: categorization (Jentzsch and Dudschig, 2009), flanker (Debener et al., 2005), and Stroop (Gehring and Fencsik, 2001) tasks. Jentzsch and Dudschig (2009) and Danielmeier and Ullsperger (2011) found that the PES effect depends on the RSI. The amplitude of this effect, defined as the difference between the mean reaction times of post-error and post-correct trials, decreases as one increases the RSI, with values going from several dozens of milliseconds to zero. For RSIs $>750-1500 \mathrm{~ms}$, PES is not observed anymore. Remarkably, the PES effect is reported in cases where the subject does not receive information on the correctness of the decision (Jentzsch and Dudschig, 2009; Danielmeier and Ullsperger, 2011; Danielmeier et al., 2011). Moreover, this effect is automatic and involuntary (Rabbitt, 2002), and is independent of error detection and the correction process, which involve other cortical areas (Rodriguez-Fornells et al., 2002). This suggests a rather low level processing origin in line with the present model.

In this section we investigate the occurrence of post-error adjustments in our model. We confront the results to empirical findings from various behavioral experiments with TAFC (and marginally also 4-AFC) protocols in which, as it is also the case in our model, there is no feedback on the correctness of the decision. We will notably discuss the model predictions comparing the results with those of Danielmeier and Ullsperger (2011) who studied the dependence of PES with respect to the RSI, as well as the relation between PES and PIA.

We studied the occurrence of the PES effect in the model with respect to the coherence level and $I_{\mathrm{CD} \text {,max }}$, at an intermediate RSI value of $500 \mathrm{~ms}$, leading to the phase diagram in Figure 9A. We find a large domain in parameter space showing PES effect (Fig. 9, red). Figure $9 B$ zooms on a value of $I_{C D \text {,max }}$ for which PES occurs 


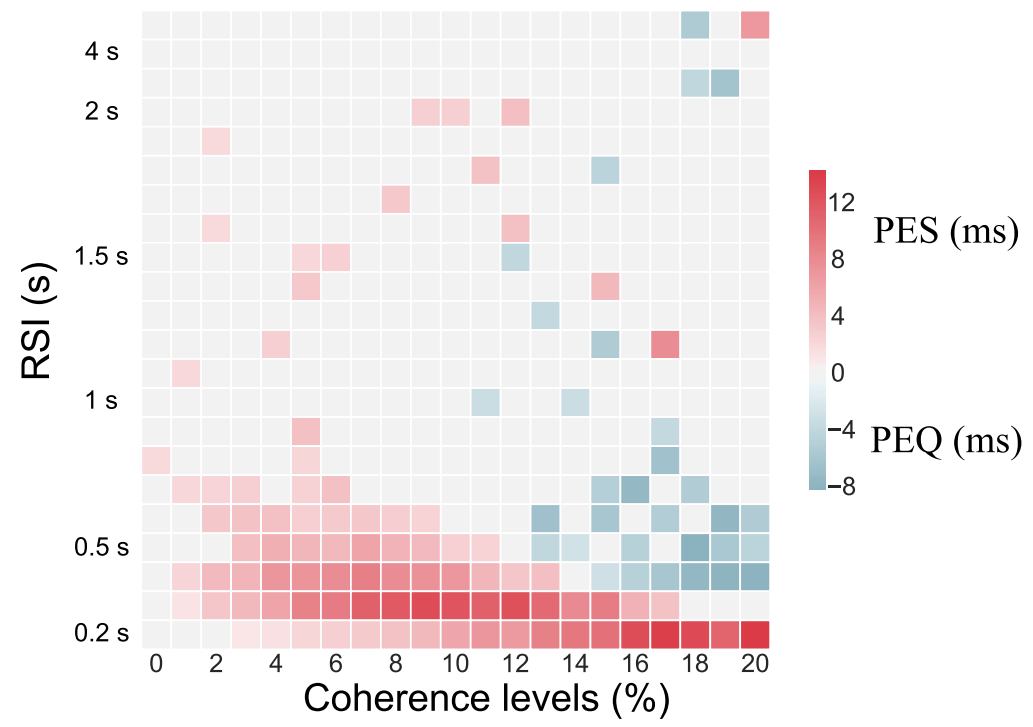

Figure 10. Post-error slowing depending on RSI. $\boldsymbol{A}$, Phase diagram of the PES effect at $I_{\mathrm{CD} \text {, } \max }=0.045 \mathrm{nA}$. The red square corresponds to regions where PES is observed, and the blue ones where PEQ is observed (the darker the color, the stronger the effect). We used a bootstrapped confidence interval to decide whether or not PES/PEQ is observed.

$\left(I_{\mathrm{CD} \text {,max }}=0.035 \mathrm{nA}\right)$. We observe that the magnitude of the PES effect goes from 0 to $10 \mathrm{~ms}$ at $c=10 \%$, hence remaining within the range of behavioral data (Jentzsch and Dudschig, 2009; Danielmeier and Ullsperger, 2011; $10-15 \mathrm{~ms}$ for a RSI of $0.5-1 \mathrm{~s}$ ). In these experiments (a flanker task with stimuli belonging to 1 of 2 opposite categories, Left or Right directions), the ambiguity level is not quantified. However, the observed error rates are found $\sim 10 \%$, which, within our model, corresponds to a coherence level of about $c=10 \%$. On the phase diagram, one can observe the variation of the PES effect with respect to the coherence level. In the region where we observe a PES effect, we find that it is enhanced under conditions when errors are infrequent. However, for large values of the coherence level, this effect cannot be observed anymore because of the absence of any error in the successive trials $(\sim 100 \%$ of correct trials). This occurrence of PES, principally at low error rates, has been found in experiments of Notebaert et al. (2009); Núñez Castellar et al. (2010), for which the authors observe PES when errors are infrequent, but not when errors are frequent. Note that these experiments are with 4-AFC tasks, but we expect the same type of properties as for TAFC tasks, and the model could easily be adapted to such cases with a neural pool specific to each one of the four categories.

The phase diagram, Figure $9 A$, also shows parameter values with no effect at all (gray), and a domain with the opposite effect, that is with reaction times faster after an error than after a correct trial (blue). We propose to call this effect post-error quickening (PEQ), as opposed to PES. As shown in Figure 9C, we find that, for a given value of $I_{\mathrm{CD} \text {, max }}$, one can have PES at low coherence level, and PEQ at high coherence level.

This PEQ effect, although much less studied, has been observed in various AFC experiments, either without feedback (Rabbitt and Rodgers, 1977; Notebaert et al., 2009; King et al., 2010) or with feedback (Purcell and Kiani, 2016), notably for fast-response regimes (Notebaert et al., 2009; King et al., 2010). The conditions for observing PEQ remain however not well established, with some contradictory results. We note that with go/no-go protocols (which are similar to AFC protocols in many respects), Hester et al. (2005) report post-error decrease in reaction times for aware errors, but not for unaware errors, whereas
Cohen et al. (2009) on the contrary reports no PEQ effect, but a larger PES effect for aware errors than for unaware errors. The fact that the model predicts PEQ in TAFC tasks at high coherence levels is more in line with the results of the fMRI experiments of Hester et al. (2005). Indeed, at high coherence levels, responses are fast and most often correct. In the rare case of an error, the subject is likely to become aware that an error has been made (Yeung and Summerfield, 2012). This thus may lead to a correlation (without causal links) between aware errors and PEQ.

We also studied the RSI dependency of the PES effects by plotting the phase diagram at $I_{\mathrm{CD} \text {, } \max }=0.045 \mathrm{nA}$ with respect to the RSI (Fig. 10). In behavioral experiments the PES effect depends strongly on the RSI. For RSIs >1000-1500 ms the observation or not of PES depends specifically on the decision task (Jentzsch and Dudschig, 2009; King et al., 2010). However, a common observation is that, whenever PES is observed, if one keeps increasing the RSI, the PES effect eventually disappears. In Figure 10, we observe that, for parameters where PES is observed at a RSI of $500 \mathrm{~ms}$, increasing the RSI leads to the weakening of the PES effect until its disappearance. At a RSI of 1000$1500 \mathrm{~ms}$ this effect is not present anymore, in agreement with experimental results (Jentzsch and Dudschig, 2009).

The variation of PEQ with respect to RSI has not been experimentally studied, as this effect is more controversial. However, our model shows that the dependence on RSI is similar to the one of PES, and predicts that when both effects exists at a same RSI value (for different coherence levels), increasing the RSI leads to the disappearance of both of them.

We note here that the set of phase diagrams that we present in this work on the various effects, Figures 9-12, provide testable behavioral predictions. As just discussed in the particular case of PES and PEQ, they predict how the effects on reaction times are or are not correlated, and in particular how they qualitatively depend on, and covary with, the coherence level or the duration of the RSI.

\section{Post-error improvement in accuracy}

PIA is another sequential effect reported in experiments (Laming, 1979a; Marco-Pallarés et al., 2008; Danielmeier and Ullsperger, 2011). PIA has been observed on different time-scales: long-term learning effects following error (Hester et al., 2005) and trial-totrial adjustments directly after commission of error responses. We only consider this latter type of PIA. The specific conditions under which PIA can be observed in behavioral experiments have not been totally understood. We investigate this effect in the specific context of our model, considering that the strength of the effect is linked to the difference in error rates between post-error and post-correct trials.

In Figure 11 we represent the phase diagram of the PIA effect with respect to coherence levels ( $x$-axis) and corollary discharge amplitude ( $y$-axis). We denote a large region of parameters for which PIA is present. We find a magnitude of the PIA effect of $\sim 2-4 \%$, which is of the same order of magnitude as in the experiments where, for RSIs in the range 500-1000 ms, it is found that 
A
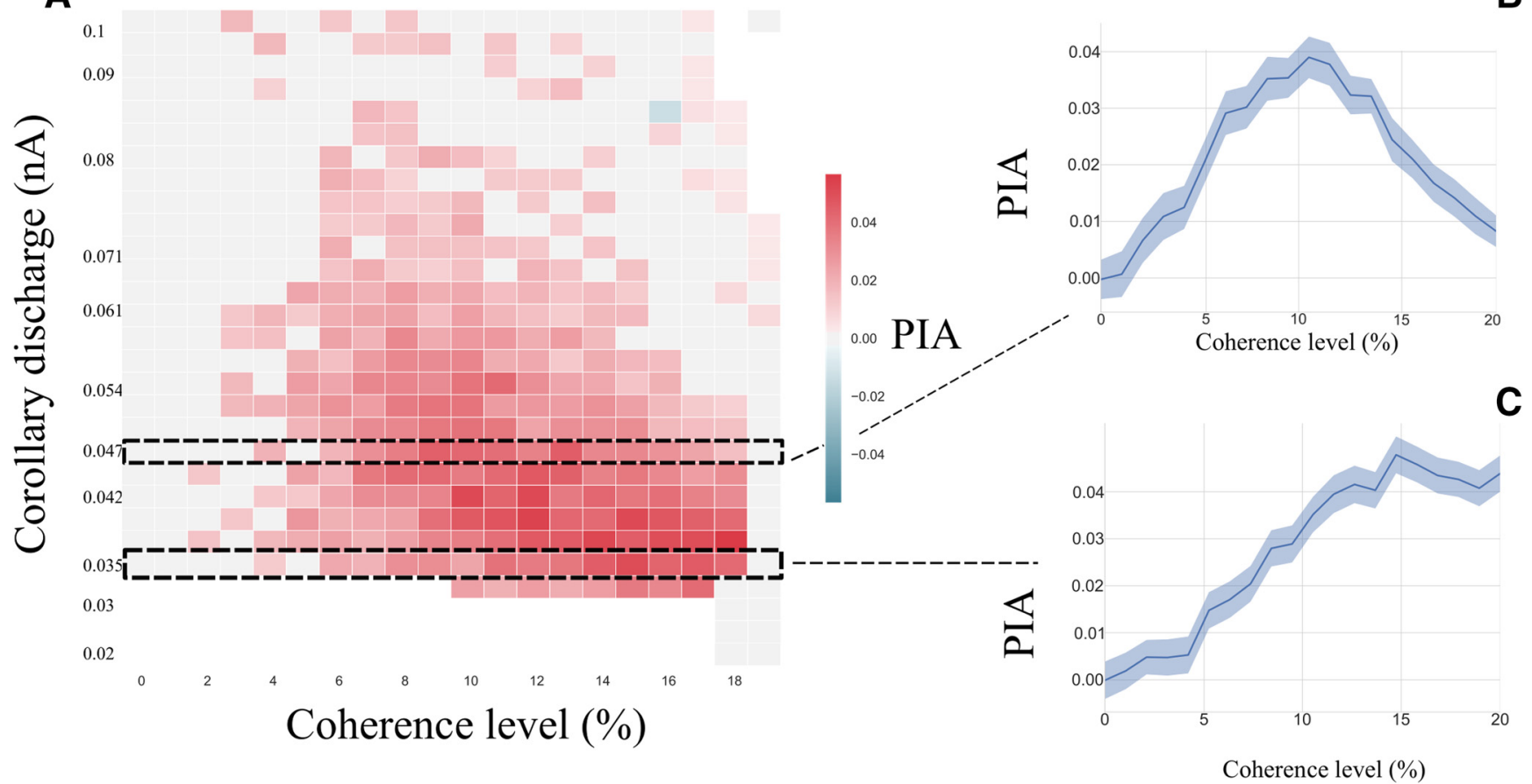

Figure 11. PIA at a RSI of $500 \mathrm{~ms}$. A, Phase diagram of the PIA effect at RSI of $500 \mathrm{~ms}$. The bottom white zone corresponds to parameters where sequential decision-making is impossible. The red square corresponds to regions where PIA is observed. The black dashed squares correspond to specific regions where $B$ and $C$ zoom. $B$, PIA effect with respect to the coherence level at $I_{C D \text {,max }}=0.047$ $\mathrm{nA}$. The light blue zone corresponds to the bootstrapped confidence interval at $95 \%$. C, PIA effect with respect to the coherence level at $I_{\mathrm{CD}, \max }=0.035 \mathrm{nA}$. The light blue zone corresponds to the bootstrapped confidence interval at $95 \%$.

post-error accuracy is improved by $\sim 3 \%$ (Jentzsch and Dudschig, 2009).

Looking at Figure 11, one sees that the PIA and PES effects append in the same region of parameters. However, if we zoom in on specific regions (Fig. $11 B, C$ ), we can notice some differences in the variation of these effects. The black dashed rectangular regions correspond to the same parameters as in Figure 9. We first note that PIA is also observed in these regions. However, we observe a decrease of PES at very large coherence (Fig. 9B), but not of PIA (Fig. 11B). Moreover the decrease of the PIA effect in Figure $9 C$ does not occur at the same values of parameters as for the PES one. It would be tempting to interpret PIA as a better accuracy resulting from taking more time for making the decision. This is not the case, because PIA does not appear uniquely in the PES region, but in the PEQ one too. In agreement with these model predictions, Danielmeier et al. (2011), in a TAFC task with color-based categories, observe that PIA can occur in the absence of PES, but that the occurrence of PES is always associated with PIA (except for 1 subject among 20, results reported by Danielmeier and Ullsperger, 2011), their Fig. 1).

In EEG experiments, Marco-Pallarés et al. (2008) find that time courses of PES and PIA seem to be dissociable as they observe post-error improvements in accuracy with longer intertrial intervals (up to $2250 \mathrm{~ms}$ ) than PES. We note that these authors consider protocols with and without stop-signals, and here we are only concerned by those without. We investigate the variation with respect to the RSI of PIA in our model (Fig. 12). We note that, for long RSIs, the PIA effect is not observed anymore. However as observed by Marco-Pallarés et al. (2008), the PIA effect occurs for longer RSIs than the PES effect (Fig. 11A). In the same way, PIA is more robust with respect to the intensity of the corollary discharge. This is corroborated by Figure 13, $A$ and $B$, which represents PES and PIA effect for a larger relaxation time, $\tau_{\mathrm{CD}}=500 \mathrm{~ms}$, hence with a stronger CD. We note that all the regimes previously observed are present, for slightly different parameter ranges. This shows that the global picture illustrated by the phase diagrams, Figures 9 and 11, is not specific to a narrow range of $I_{\mathrm{CD} \text {,max }}$ and $\tau_{\mathrm{CD}}$ values.

Verguts et al. (2011) find that PIA and PES seem to happen independently, suggesting that at least two post-error processes takes place in parallel. An important outcome of our analysis is to show that PIA and PES effects can both result from the same underlying dynamics. In addition, in the parameters domain where they both occur, we find that the variations of these effects with respect to the coherence levels are indeed uncorrelated (Pearson correlation test: RSI of $500 \mathrm{~ms}$ and $I_{\mathrm{CD}}=0.035 \mathrm{nA}, p=$ $0.58, I_{\mathrm{CD}}=0.05, p=0.79$ and $I_{\mathrm{CD}}=0.1 \mathrm{nA}, p=0.25$; RSI of 2000 $\mathrm{ms}$ and $\left.I_{\mathrm{CD}}=0.035 \mathrm{nA}, p=0.37\right)$. This non-correlation highlights the complexity of such post-error adjustments, as explored by Verguts et al. (2011).

To gain more insights into the PIA effect, we study the discrimination threshold following an error or a success, with respect to the RSI (Fig. 12B-D). In Figure $12 B$ we represent the distribution of the discrimination threshold for $I_{\mathrm{CD} \text {, max }}=0.035$ $\mathrm{nA}$ and a RSI of $500 \mathrm{~ms}$. For these parameters, the distributions for the post-error and post-success cases are highly different (Smirnov-Kolmogorov test: $p<10^{-20}$ ). If one increases the RSI (Fig. 12C, $1000 \mathrm{~ms}, D, 1500 \mathrm{~ms}$ ), this difference disappears (Smirnov-Kolmogorov test: $p=0.038$ and $p=0.4$, respectively). However, we note that the model predicts a wider distribution of the discrimination threshold after an error than after a correct trial, independently of the presence of the PIA effect. This might result from the wider distribution in the neural (or synaptic) activities after an error that we discuss $n$ the next section. To 


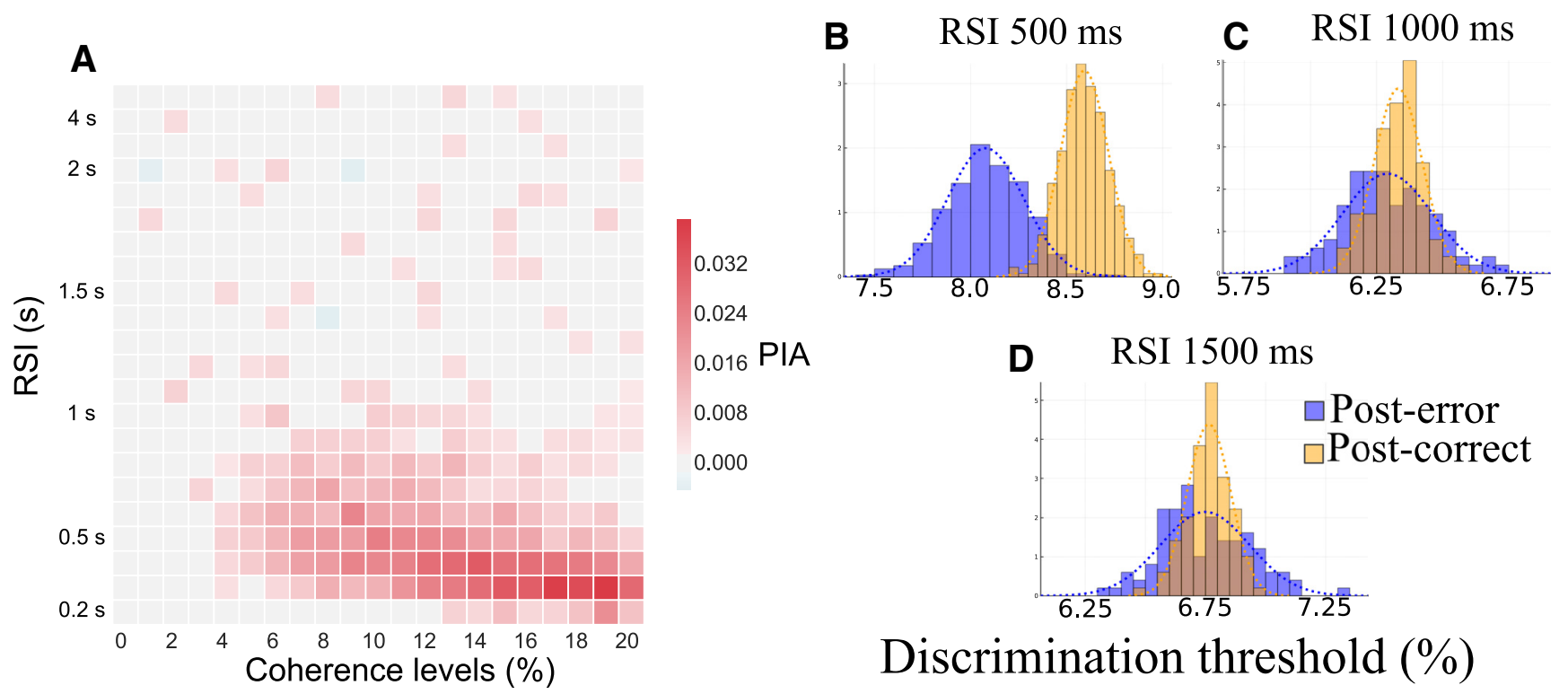

Figure 12. Post-error improvement in accuracy depending on RSI. $\boldsymbol{A}$, Phase diagram of the PIA effect at $I_{\mathrm{CD}, \max }=0.045 \mathrm{nA}$. The red square corresponds to regions where PIA is observed. $\boldsymbol{B}-\boldsymbol{D}$, Distribution of the discrimination threshold for three values of RSI (500, 1000, $1500 \mathrm{~ms}$, respectively). In yellow we represent the histogram of the post-correct trials, and in blue the post-error ones. The dashed curves of the corresponding color corresponds to the cumulative functions of these distributions. The $C D$ is $I_{C D, \max }=0.035 \mathrm{nA}$.

A

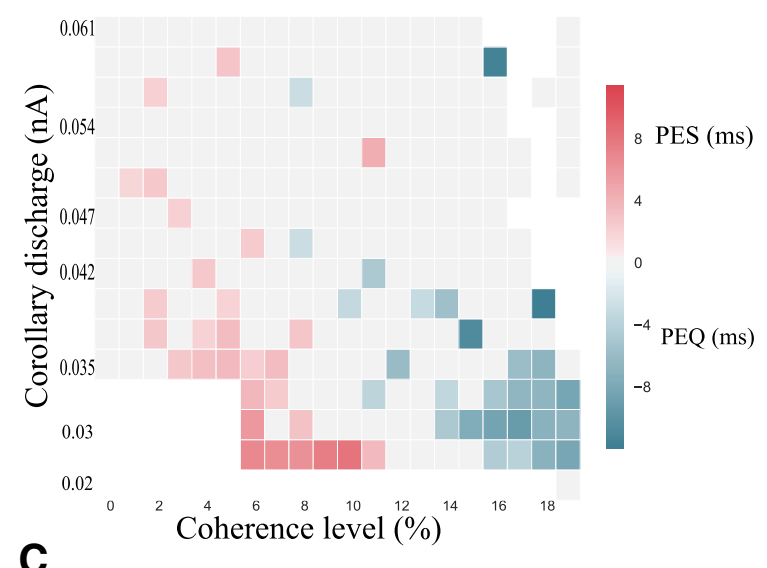

C

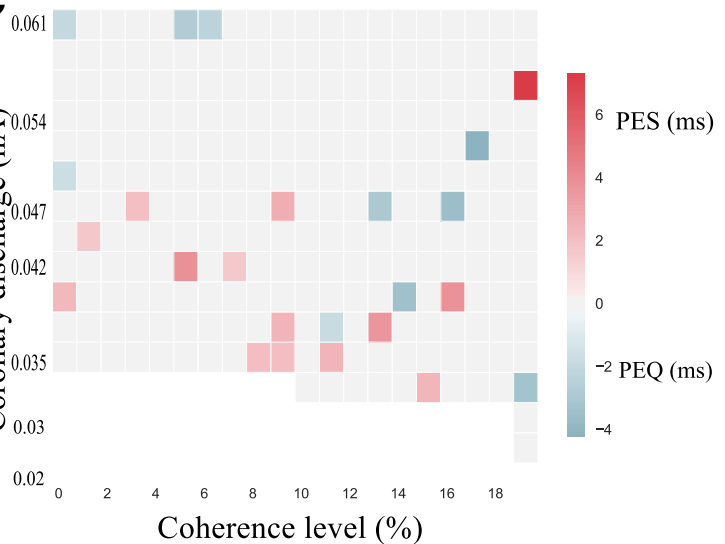

B
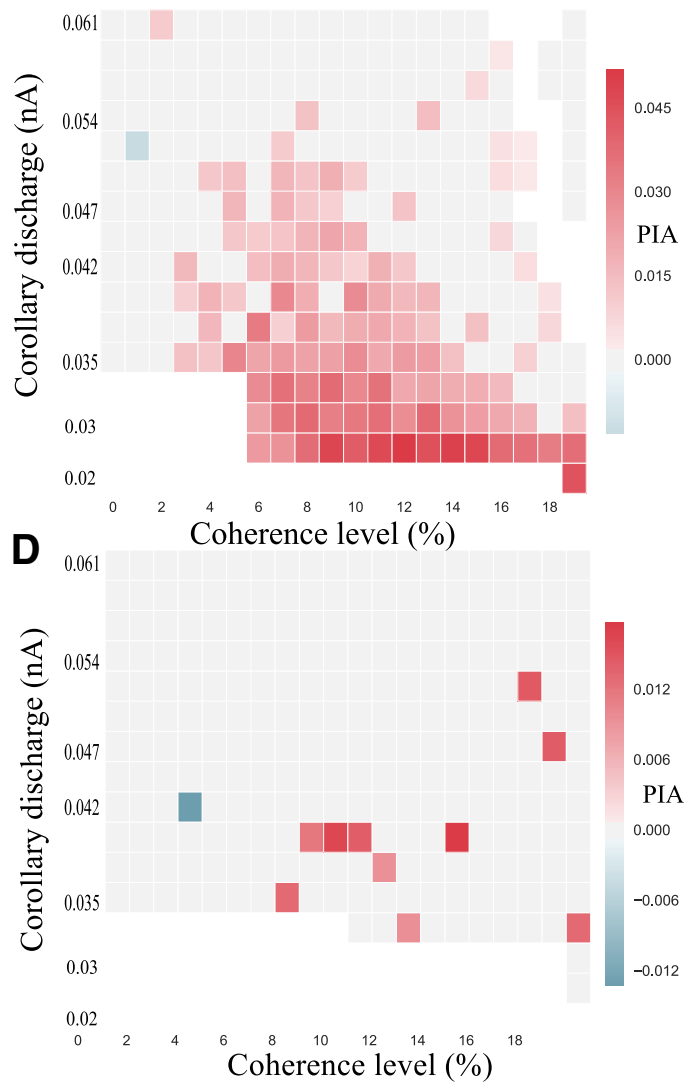

Figure 13. Post-error adjustments at $\tau_{C D}=500 \mathrm{~ms}(\boldsymbol{A}, \boldsymbol{B})$, and second-order post-error adjustments $(\boldsymbol{C}, \boldsymbol{D})$. $\boldsymbol{A}$, Phase diagram of the PES effect. We used a bootstrapped confidence interval to decide whether or not PES (or PEQ) is observed. $\boldsymbol{B}$, Phase diagram of the PIA effect. The observation of post-error adjustments is highly impacted with the value of $\tau_{C D}$, because we do not observe PES for the same range of parameters. C, Phase diagram of the PES effect at the $n+2$ trial. D, Phase diagram of the PIA effect at the $n+2$ trial. One sees rare isolated red squares, indicating the absence of any systematic effect. For all panels, simulations with a RSI of $500 \mathrm{~ms}$, other parameters as in Table 1. Color-code as in Figure 9. 

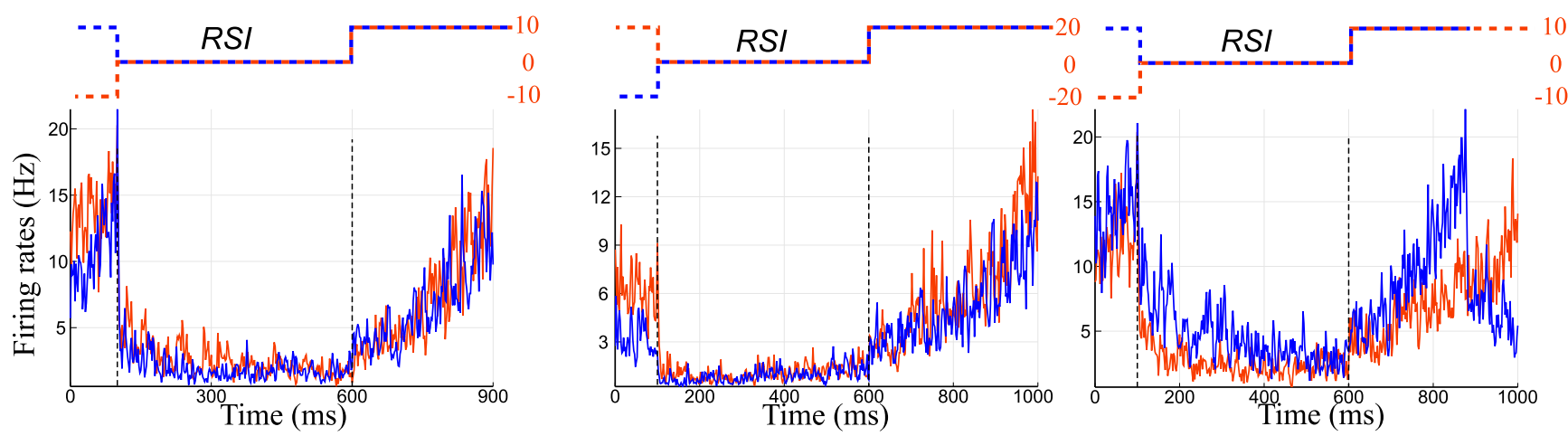

----- Stimulus intensity for post-error case _- Post-Error firing rates (population L)

----- Stimulus intensity for post-correct case _ P Post-Correct firing rates (population L)

Figure 14. Neural activities of individual trials. $\boldsymbol{A}$, Dynamics for individual trials for the winning populations of the next trial: in blue the post-correct case and in red the post-error one. The dashed lines represent the coherence of the stimuli with respect to time. In blue we represent the post-correct case, and in red the post-error one. The parameters are set to a region without $P E S$ or $P E Q$ effects $\left(I_{C D}=0.047 \mathrm{nA}\right.$ and $\left.c= \pm 10 \%\right)$. $B$, Dynamics in the region of PEQ $\left(I_{C D}=0.047 \mathrm{nA}\right.$ and $\left.c= \pm 20 \%\right)$. On this trial we can notice that the post-error dynamics is faster than the post-correct one. $C$, The parameters are set to the PES region ( $I_{C D}=0.035 \mathrm{nA}$ and $c= \pm 10 \%$ ). The post-correct dynamics (in blue) reaches the threshold sooner than the post-error one (in red).

our knowledge, this effect has not been studied in behavioral experiments.

\section{Dynamical analysis}

In this section we analyze the PES and PEQ effects in terms of neural dynamics. First of all, we represent and discuss the dynamics on individual trials for the three regions of parameters: with neither PES nor PEQ effects, with PES effect, and with PEQ effect (Fig. 14). We observe the dynamics for post-error and postcorrect trials during the relaxation period following a decision and during the presentation of the next stimulus. Already on individual trials we notice differences between the regions. Figure $14 A$ represents a trial in the region without PES or PEQ. The post-error/correct dynamics are indistinguishable. Hence we do not observe any differences in the reaction times. Looking at a trial in the PEQ region (Fig. 14B), we notice that the population $L$ (the winning one for the second stimulus) for the post-error case seems a bit higher in activity than for the post-correct case. This leads to the post-error quickening effect, as the post-error (orange) curve will reach the threshold sooner than the post-correct (blue) one. Finally, Figure $14 C$ represents individual trials for parameters in the PES region. In the phase diagram (Fig. 9) the effect was more pronounced than PEQ, thus it is more pronounced on the dynamics too. During the relaxation, and the presentation of the next stimulus, the post-correct dynamics (blue curve) for population $L$ (the winning one for the second stimulus) is higher than the post-error one. As we can observe this leads to a faster decision time for the post-correct trial than for the post-error one.

We show now that the dynamics explains the three effects PES, PEQ and PIA. We provide in Figures $16-18$ a semiqualitative and semiquantitative analysis of the dynamics of the synaptic activities in the phase plane of the system, for several parameter regimes. Here again, the analysis is easier working on the synaptic activities. This can be seen by considering Figure 15 on which we represent the mean firing rate and synaptic activity of the winning population in the PES case. Because of the range of variation of the firing rates, and the intrinsic noise of the system, it is hard to observe a difference between the neural activities. However, if we compute this difference (Fig. 15, inset) we note the following. At the beginning of the next trial, the difference between the posterror and post-correct firing rates is significantly below zero, hence the reaction time will be shorter for post-correct than for post-error trials. We find the same behavior for the synaptic activities (Fig. 15B), but much less noisy, as expected from the discussion in Materials and Methods.

\section{PES effect}

We now detail the analysis of the PES effect (and of the concomitant PIA effect) based on Figure 16. Let us first explain how each panel is done. Without loss of generality, we assume that the last decision made is $R$. Repeated and Alternated cases thus correspond to next trial decisions $R$ and $L$, respectively. The $x$ - and $y$-axes are the synaptic activities $S_{L}$ and $S_{R}$, respectively; hence, the losing and winning populations for the first trial.

On the left, we represent with dashed lines the average dynamics during the relaxation period, that is from the decision time for the previous stimulus to the onset of the next stimulus. This allows to identify clearly the typical neural states at the end of the relaxation period. The average is done over post-error (respectively, post-correct) trajectories sharing a same state at the time of the last decision. The choice of these two initial states is based on the following remark. A typical trial with a correct decision will lead, at the time of decision, to losing and winning populations with highly different activity rates, hence a neural activity, and thus a synaptic activity $S_{L}$, far from the threshold value. On the contrary, a typical error trial will show a losing activity not far from the threshold; this can also be observed in the study byWong et al. (2007, their Fig. 4B). We can thus represent postcorrect trials, respectively post-error trials, by dynamics with initial states having a rather small, respectively large, value of $S_{L}$ (and in both cases the first trial winning population $S_{R}$ at threshold value).

We then represent with a continuous line the average trajectory following the onset of the next stimulus. We observe this dynamics during the same time for post-error and postcorrect cases, as if there were no decision threshold, to compare the dynamics of post-error and post-correct cases for the 
A

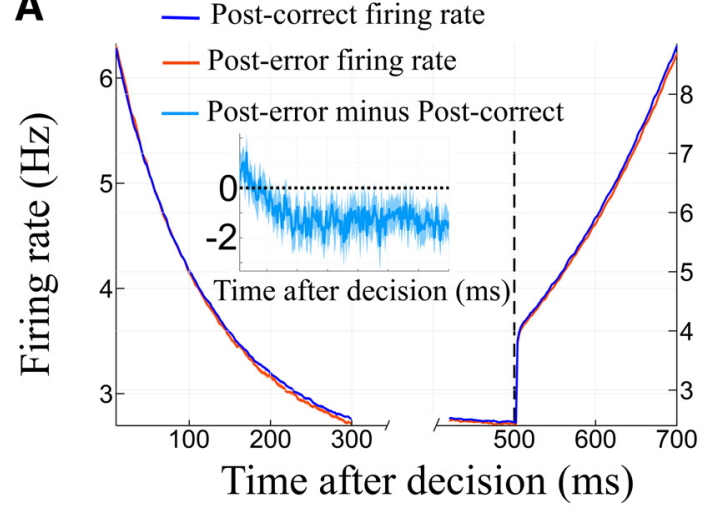

B

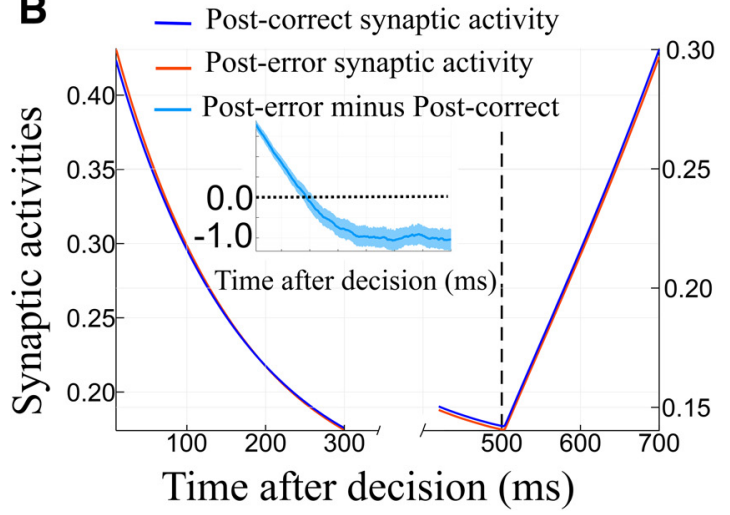

Figure 15. Mean firing rates of the winning population. $A$, Mean firing rates for the winning populations of the next trial: in blue the post-correct case and in orange the post-error one. The ribbons represent the $95 \%$ confidence interval on 25 simulations (bootstrap method). The left-axis represents the relaxation of the dynamics. The right-axis is for the beginning of the next stimulus. The parameters are set to a region with PES effects $\left(I_{C D}=0.035 \mathrm{nA}\right.$ and $\left.c= \pm 10\right)$. Inset with the light blue curve is the difference between the post-error firing rates and the post-correct with respect to time (percentage). The ribbon stands for the $95 \%$ confidence interval. As expected, this difference is negative. Hence the post-correct dynamics is faster and crosses sooner the threshold. This leads to the PES effect. $\boldsymbol{B}$, Mean synaptic activities for the winning populations of the next trial: in blue the post-correct case and in orange the post-error one. Inset with the light blue curve is the difference between the post-error synaptic activities and the post-correct with respect to time (percentage).

A

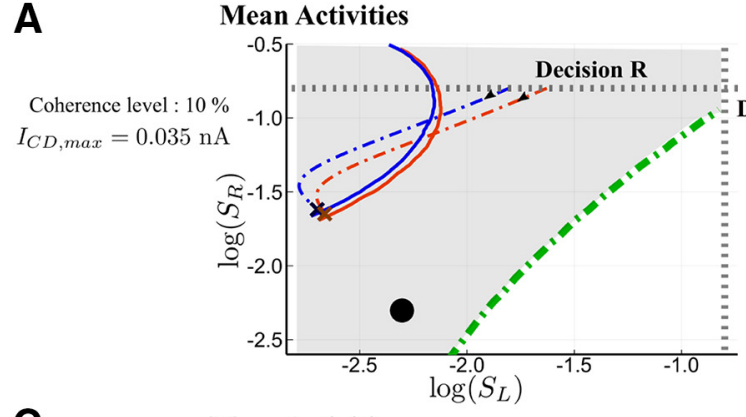

C

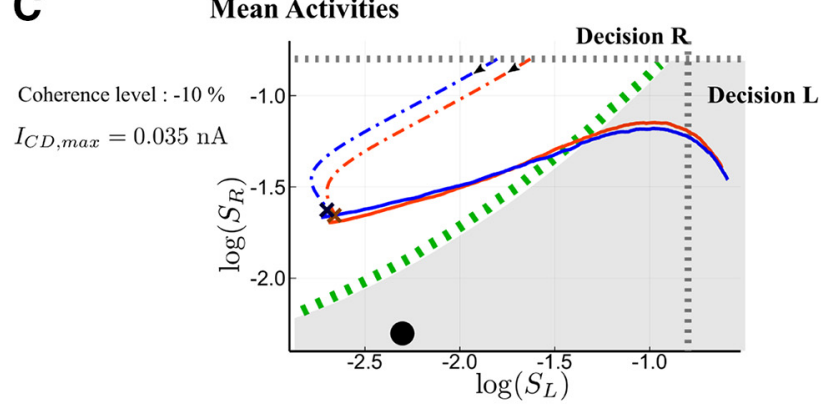

B

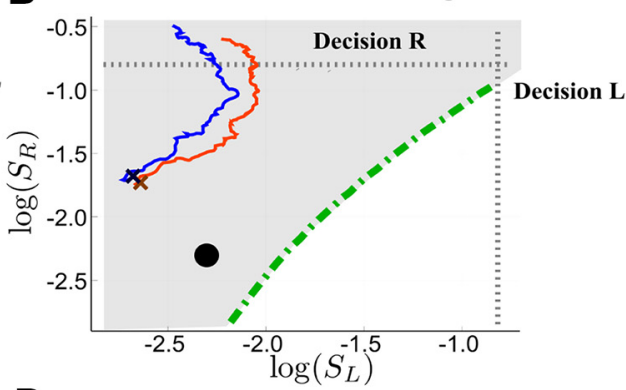

D
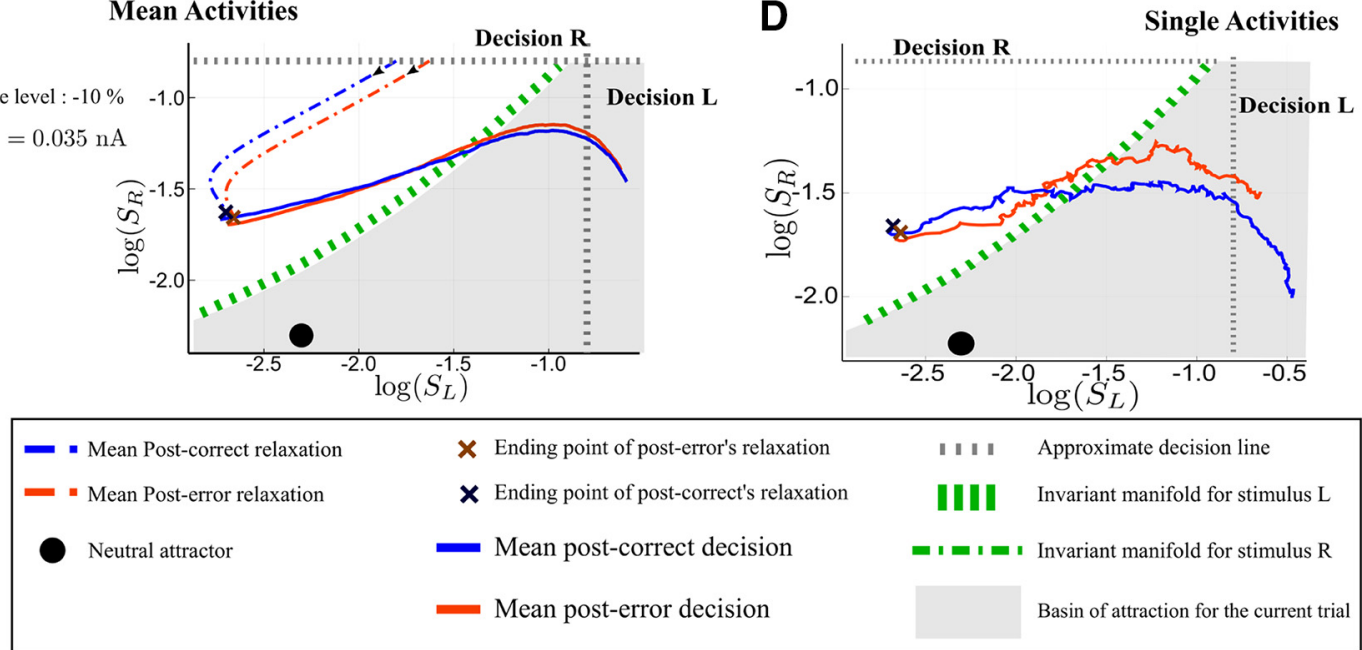

Figure 16. Analysis of the post-error trajectories for the PES regime. Phase-plane trajectories (in log-log plot, for ease of viewing) of the post-correct and post-error trials. We consider that the previous decision was decision R. The black filled circle shows the neutral attractor state (during the relaxation period). During the presentation of the next stimulus, the attractors and basins of attraction change (gray area and the green dashed lines). $A, B, P E S$ and PIA regime $\left(c=10 \%\right.$ and $\left.I_{C D, \max }=0.035 \mathrm{nA}\right)$ in the repeated case. The blue color codes for post-correct trials, and the red one for post-error. $\boldsymbol{A}$, Average dynamics; $\boldsymbol{B}$, single trajectories during the next trial. $\boldsymbol{C}, \boldsymbol{D}$, Regime with PES and PIA in the alternated case $\left(\boldsymbol{C}=-10 \%\right.$ and $\left.I_{\mathrm{CD}, \max }=0.035 \mathrm{nA}\right)$. The dynamics after the relaxation is followed during $400 \mathrm{~ms}$ for repeated and $800 \mathrm{~ms}$ for alternated case, as if there were no decision threshold. The actual decision occurs at the crossing of the dashed gray line, indicating the threshold.

same duration of time. Decision actually occurs when the trajectory crosses the decision line (dashed gray line); this is approximate: because of the noise, there is no one to one correspondence between a neural activity reaching the decision threshold and a particular value of the associated synaptic activity. Having all the trajectories plotted for the same dura- tion (and not only until the decision time) allows to visually compare the associated reaction times.

On the right, we represent typical trajectories during the presentation of the next stimulus. The black dot on every panel gives the location of the neutral attractor that exists during the relaxation dynamics. The basins of attractions that are represented are 
A

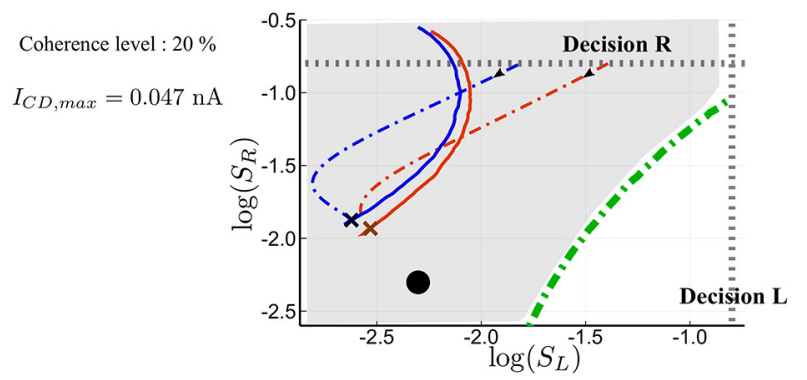

C

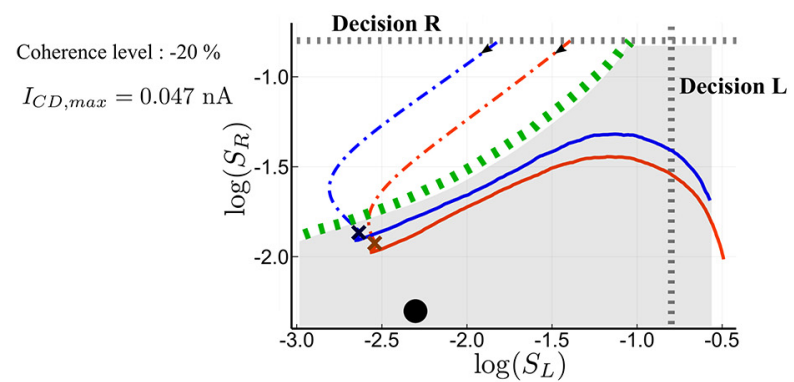

B

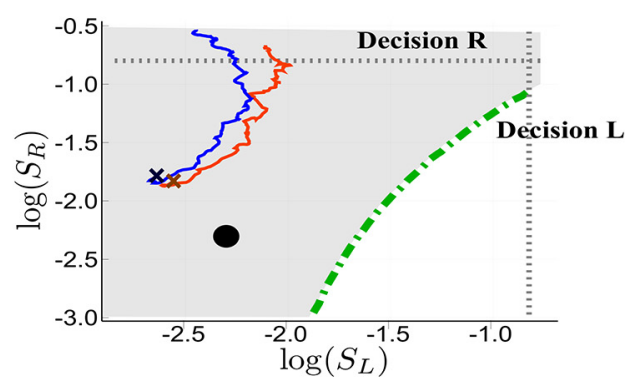

D

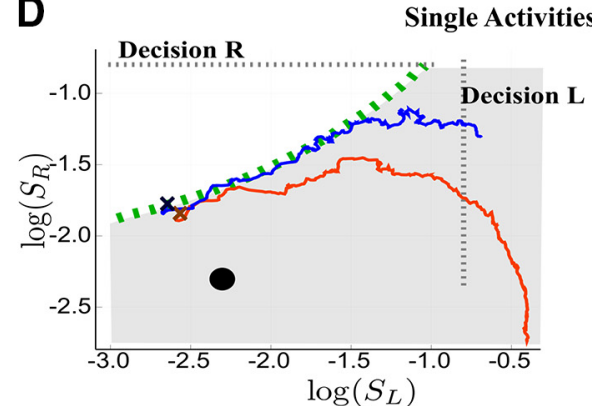

$$
\begin{aligned}
& \text { - Mean Post-correct relaxation } \\
& \text { - Mean Post-error relaxation } \\
& \text { Neutral attractor }
\end{aligned}
$$

$X$ Ending point of post-error's relaxation

X Ending point of post-correct's relaxation

Mean post-correct decision

Mean post-error decision
II If Approximate decision line

Invariant manifold for stimulus $\mathrm{L}$

ㅂ ı เ ・ Invariant manifold for stimulus R

Basin of attraction for the current tria

Figure 17. Analysis of the post-error trajectories in the PEQ regime. Phase-plane trajectories (in log-log plot, for ease of viewing) of the post-correct and post-error trials (same as Fig. 16 in the $P E Q$ case). We consider that the previous decision was $R$. The black filled circle shows the neutral attractor state (during the relaxation period). During the presentation of the next stimulus, the attractors and basins of attraction change (gray area and the green dashed line). $A, B, \mathrm{PEQ}$ and PIA regime $\left(c=20 \%\right.$ and $\left.I_{\mathrm{CD} \text {, max }}=0.047 \mathrm{nA}\right)$. The blue color codes for post-correct trials, and the red one for post-error. The plain lines represent mean dynamics for $(\boldsymbol{A})$ or single dynamics $(\boldsymbol{B})$. $\boldsymbol{C}, \boldsymbol{D}$, Regime with PEQ and PIA in the alternated case $\left(\boldsymbol{C}=-20 \%\right.$ and $\left.I_{C D, \max }=0.047 \mathrm{nA}\right)$. The post-error relaxation already lies within the alternated basin of attraction. For alternated trials, the dynamics needs to cross the invariant manifold (green dashed line), which denotes the boundary between the basins of attraction. The dynamics is followed during $400 \mathrm{~ms}$ for repeated and $800 \mathrm{~ms}$ for alternated case, as if there were no decision threshold. The actual decision occurs at the crossing of the dashed gray line, indicating the threshold.

the ones associated with the attractors, $L$ and $R$, of the dynamics induced by the onset of the next stimulus. Be reminded that these attractors are different from the ones associated to the dynamics during the relaxation period.

We can now analyze the dynamics. In the repeated case (Fig. $16 A, B$ ), at the end of the relaxation (that is at the onset of the next stimulus), both post-correct and post-error trials lie into the correct basin of attraction. Hence, the error rates for these trials are similar. However, the neural states reached at the end of the relaxations are different. Compared with the post-error trial, the post-correct state is closer to the boundary of the new attractor associated to decision $R$, and the corresponding decision will thus be faster. In the alternate case (Fig. 16C,D), the states reached at the end of the relaxation period do not lie within the correct basin of attraction. During the decision-making dynamics, the trajectory needs to cross the boundary between the two basins of attraction. The post-correct trials leading to an alternate decision have rather straight dynamics across the boundary, leading to relatively fast decision times. In contrast, the states at the onset of the stimulus of the post-error trials are closer to the boundary so that the corresponding trajectories cross with a smaller angle with respect to the basin boundary. This leads to longer reaction times, hence the PES effect. It would be interesting to have electrophysiological data with which the model predictions could be directly compared. However, in a typical experiment on monkeys, a feed- back on the correctness of the decision is given, because the animal learns the task thanks to a reward-based protocol. Nevertheless, we note that, in the random-dot experiments on monkeys by Purcell and Kiani (2016), the authors find a higher buildup rate of the neural activity for post-correct trials than for post-error trials (Purcell and Kiani (2016), their Fig. 6). Within our framework, this can be understood as trajectories that cross the basin boundary more quickly for post-correct trials, in accordance with our model's predictions. This suggests that the observed difference in buildup rates may not result from some mechanism making use of the information on the correctness of the decision, but rather from the nonlinear dynamics discussed here.

The PIA is understood from the same analysis as for the PES effect. For specific realizations of the noise that lead to error trials, the post-error trials dynamics is closer to the boundary. Thus it has a higher probability to fall on the other side of the basin of attraction. Hence, the error rates are lower for post-error trials than post-correct trials.

\section{PEQ effect}

The PEQ effect can be understood from the same kind of analysis, based here on Figure 17 (analogous for the PEQ effect to Fig. 16 for the PES effect). As seen previously, the PEQ effect occurs mostly at high level of coherence. We consider first the repeated 
A

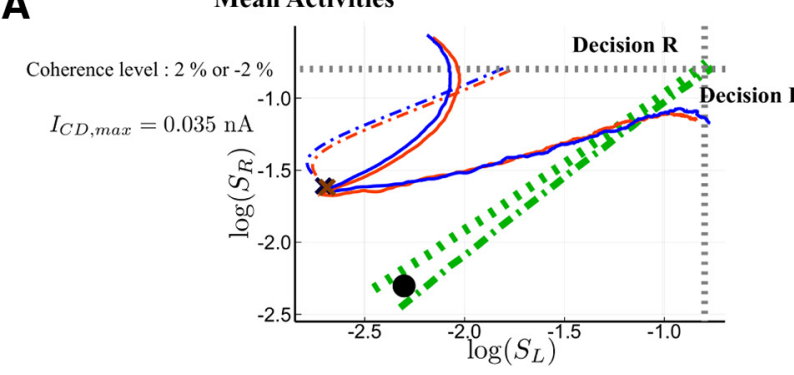

C

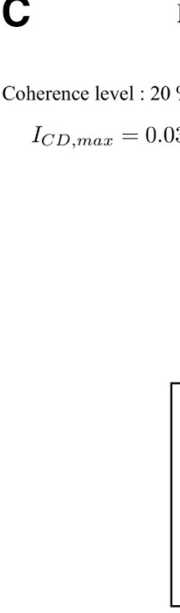

\section{Mean Activities}

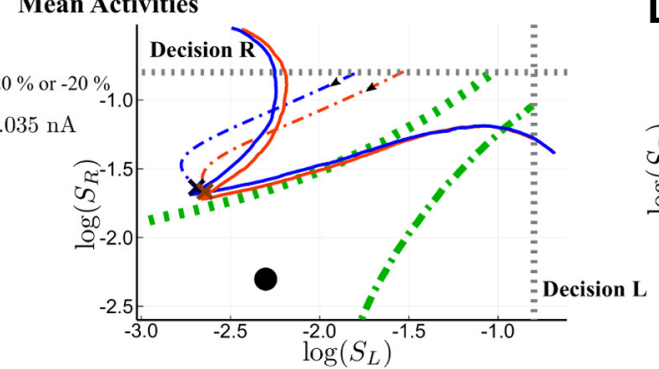

- Mean Post-correct relaxation

- " Mean Post-error relaxation

Neutral attractor
B

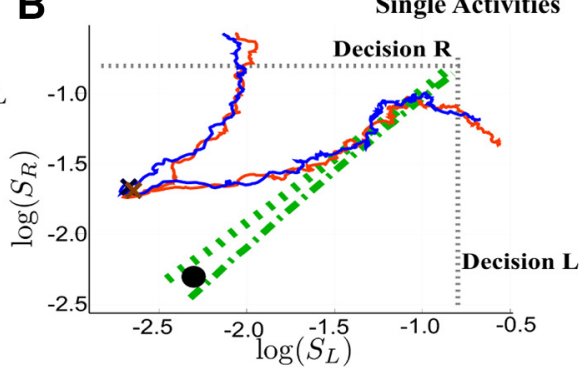

$x$ Ending point of post-error's relaxation

X Ending point of post-correct's relaxation

Mean post-correct decision

Mean post-error decision

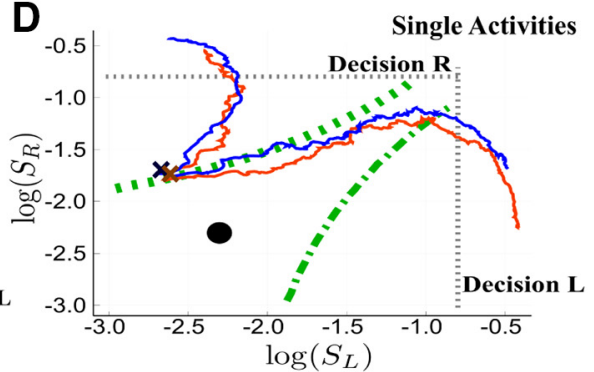

Figure 18. Analysis of the post-error trajectories in the regime with neither PES nor PEQ effect Phase-plane trajectories (in log-log plot, for ease of viewing) of the post-correct and post-error trials. We consider that the previous decision was decision R. The black filled circle shows the neutral attractor state (during the relaxation period). During the presentation of the next stimulus, the attractors and basins of attraction change (gray area and the green dashed lines). $\boldsymbol{A}$, (mean dynamics) and $\boldsymbol{B}$ (single dynamics), Regime without PES or PIA ( $\boldsymbol{C}= \pm 2 \%$ and $I_{\mathrm{CD} \text {, max }}=0.035 \mathrm{nA}$ ). We show both the alternated and the repeated case, with the corresponding basins of attraction. The blue color codes for post-correct trials, and the red one for post-error. For alternated trials, the dynamics needs to cross the invariant manifold (green dashed line), which denotes the boundary between the basins of attraction. $\boldsymbol{C}$ (mean dynamics) and $\boldsymbol{D}$ (single dynamics), Regime with PIA but without PES $\left(c= \pm 20 \%\right.$ and $I_{C D, \max }=0.035 \mathrm{nA}$ ). The dynamics is followed during $400 \mathrm{~ms}$ for repeated and 800 ms for alternated case, as if there were no decision threshold. The actual decision occurs at the crossing of the dashed gray line, indicating the threshold.

case (Fig. 17 A, B). Because the coherence level is high, at the end of the relaxation period, both post-correct and post-error trials lie within the correct basin of attraction, far from the basin boundary. The reaction times and error rates of post-correct and post-error trials for repeated decisions are thus similar.

In contrast, the alternated case (Fig. 17C,D) exhibits both the PIA and the PEQ effects. The post-error's end of relaxation now is inside the basin of attraction of the alternated choice. Hence, the error rate will be lower than when the ending point is outside this region (postcorrect trials begin at the boundary of the basin of attraction). Moreover, the post-correct trials dynamics have to cross the boundary. Hence they are closer to the manifold, which lead to slower dynamics, whereas the post-error dynamics can directly reach the new attractor state. This analysis explains why the decreasing of PES and PIA do not occur at the same coherence level too. Indeed the decreasing of PIA occurs when the ending point of the post-error relaxation crosses the boundary, whereas the post-correct ending point remains into the same basin of attraction. For the PES effect to decrease, the dynamics for both cases just need to be closer to the boundary and not necessarily on the opposite side. Hence the decrease of the PES effect occurs at lower coherence than the PIA one.

Here we have seen that the occurrence of the PEQ effect depends on some very specific and fragile feature, the crossing or not of a basin boundary. The conditions for observing the effect are thus likely to vary from individual to individual, and from experiment to experiment. This may explain why the experimental results about the PEQ effect remain controversial.
In Figure 18, $A$ and $B$, we investigate the parameter regime, at low coherence level, for which there is no effect; neither PES, nor PEQ or PIA. The post-error and post-correct dynamics are very similar and lead to the same relaxation ending point, far from the basin boundary. Finally, in Figure 18, $C$ and $D$, we consider the parameter regime, at high coherence level, with only the PIA effect. Here the relaxations of post-error and post-correct trials are different. However, as for the PEQ effect, at high coherence level both dynamics will be fast. For alternated trials, none of the two ending points are in the correct basin of attraction.

As discussed for the PES effect, electrophysiological data only exist for experiments with feedback on the correctness of the decision. In experiments on monkeys, Purcell and Kiani (2016) obtain puzzling results for what concerns the PEQ effect. They observe an important difference in baseline activities for post-correct and posterror trials, which is not well accounted for either by their DDM analysis or by our model. However, in terms of neural dynamics, this observed difference in the level of neural activities obviously implies that the dynamical states are different at the time of the onset of the stimulus, a fact in agreement with our model's predictions. One may wonder if the separation in baseline activities, and not just in starting points, could be a consequence of the feedback.

Correlating post-error effects with the activity distributions at the previous decision

To go beyond the above analysis on the post-error adjustments (PES, PEQ, and PIA effects), we analyze the respective influence 
of the winning and losing population levels of activity at the time of the previous decision, onto the decision at the next trial. This will first confirm the previous analysis, but also provide more insights on the specificity of the two opposite effects, PES and PEQ.

The mean activity, at the time of the decision, of the winning population is indistinguishable between post-correct and post-error trials [unequal variance (Welch) test: fail to reject, $p=0.16$ at RSI of $500 \mathrm{~ms}$; fail to reject, $p=0.87$ at RSI of $2000 \mathrm{~ms}$ ]. However, for short RSIs (corresponding to PES regime) the mean synaptic activities, at the time of the decision, of the losing population are different for post-correct and post-error trials are different for post-correct and post-error trials [unequal variance (Welch) test: reject, $p=2.7 \times 10^{-20}$ at RSI of $500 \mathrm{~ms}$; fail to reject, $p=0.57$ at RSI of $2000 \mathrm{~ms}$ ].

To gain more insight, we plot in Figure 19 the amplitude of the PES effect with respect to the interpercentile range of the distribution of the synaptic activities of the winning and losing populations at the time of the previous decision. We note that when PES occurs, the higher the activity of the losing population at the time of decision, the stronger this effect will be. The influence of the winning population is observed, although in an opposite way. When PES occurs these effects are correlated (dark blue: Pearson correlation: $r=-0.98, p=2.6 \times 10^{-7}$; medium blue: $r=-0.98$ and $p=$ $\left.9.5 \times 10^{-7}\right)$, in the sense that the variations with respect to the interpercentile of the winning and losing population are correlated. These observations are consistent with the analysis of the PES phase-plane trajectories. Indeed, the higher the losing population activity is, the closer to the invariant manifold the state at the end of the relaxation period will be. Hence, the effect will be stronger as it becomes easier (more likely) to cross the boundary.

However, we observe in Figure 19, $A$ and $C$, a different behavior for the PEQ effect: there is an almost constant value of the PEQ effect with respect to the interpercentiles of the distributions of the winning and losing populations activities. This is explained by the fact that, at the end of the relaxation, if the category of the next stimulus is the opposite of the previous decision, the network state finds itself within the (correct) associated basin of attraction, but very close to the boundary. This is true whatever the correctness of the previous decision. However, the postcorrect case will lead to an even closer location from the basin boundary. The nonlinearity of the dynamics near the basin boundary will strongly amplify the small difference between post-correct and post-error ending point. The PEQ effect will thus not be correlated with the size of this difference.

For what concerns the PIA effect, we observe in Figure 19, C and $D$, a similar dependency in the synaptic activities as for the PES effect, with a stronger effect for high activities of the losing population. This corroborates the above phase plane analysis of the trajectories (Fig. 13). Indeed, the PES and PIA effects both depend on the position of the relaxation in the phase plane. Being closer to the boundary (high activity of the losing population) leads to a smaller error rate in the next trial.

From the above analysis, a prediction of the model is that, whenever there are PES or PIA effects, the mean activity of the losing population is different for correct and error decisions. Moreover, this level of activity is correlated with the amplitude of the post-error adjustment effect. This can be seen in Figure 19, $A$ and $B$. In this figure we present the quantiles of the synaptic activities. The results would be similar, but much more noisy, for the firing rates. We expect that this prediction can be tested in experiments by measuring the correlation between the amplitude of the PES (or PIA) effect, and the difference in mean activities of the losing neural population (difference between post-error and post-correct trials).

\section{Discussion}

We have shown that, without fine tuning of parameters, an attractor neural network accounts, qualitatively and with the correct orders of magnitude, for sequential effects and post-error adjustments reported in TAFC experiments in the absence of feedback about the correctness of the decision.

We provide evidence that these effects all result from the same intrinsic properties of the nonlinear neural dynamics. We present in Figure 20 a schematic diagram of the occurrence of the effects depending on the parameters, even though this does not exhaust the richness of the systems behavior as discussed in this paper. Our results suggest to test experimentally this general picture, and more precisely what is predicted by the phase diagrams, Figures $9-12$. In particular it would be interesting to test the occurrence of post-error quickening at large coherence level or the variations of post-error adjustments with respect to coherence levels.

\section{Explanations for PES}

Several cognitive explanations of PES effects have been proposed (Rabbitt and Rodgers, 1977; Laming, 1979b; Notebaert et al., 2009).

In particular, these effects have been discussed in the framework of DDMs (Dutilh et al., 2012; Goldfarb et al., 2012; Purcell and Kiani, 2016). Dutilh et al. (2012), in experiments without feedback about the correctness of the decision, and Purcell and Kiani (2016), but in experiments with feedback, show that posterror and post-correct trials can be fitted by DDMs with different sets of parameter values for post-error and post-correct trials. In addition, Dutilh et al. (2012) argue that the modification of the decision threshold within the DDM framework, would corre- 


\section{Distance to the boundary in the alternated case}

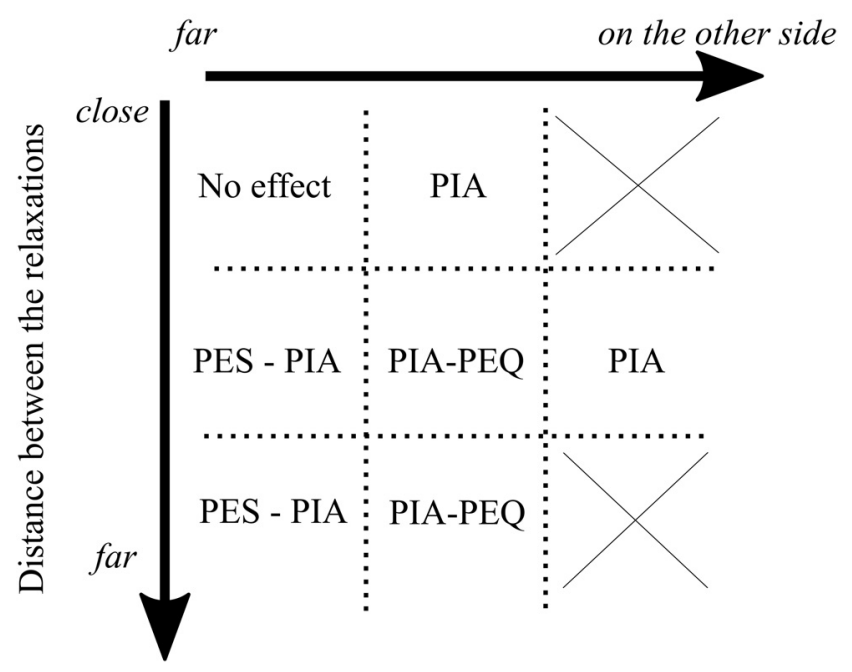

Figure 20. Schematic diagram of the post-error adjustments observations. The $x$-axis represents the distance between the ending state of the relaxations and the boundary of the following basins of attraction. It goes from "the ending states are far away from the boundary" to "both ending states are in correct basin of attraction". The $y$-axis corresponds to the distance between the post-error and post-correct relaxations. The crosses denote regions that are not relevant, or inside which the network do not commit errors.

spond to the hypothesis of increased response caution, the decision becoming more cautious after an error. Yet, the neural correlates, which would determine the threshold or the starting point remain obscure, especially in the absence of feedback on the correctness of the trial.

Within the attractor network framework considered here, the PES and PEQ effects are explained thanks to an in-depth analysis of the neural dynamics. We have shown that the location of the dynamical state at the end of the relaxation period (end of the RSI), with respect to the basins of attraction of the attractors induced by the next stimulus, depends on what occurred at the previous trial. The fact that we have different properties, e.g., for post-correct and posterror trials, for a same set of parameter values, is a result of the nonlinear dynamics which amplifies the difference in ending points of the relaxation. This cannot be obtained within the DDM framework (without the addition of other mechanisms) because, in a DDM, the state reached at the time of a decision is identical for an error and a correct trial. An additional outcome of the analysis is that, for a given set of parameter values, different regimes (PES, PEQ, or no effect) may be observed depending on the coherence level of the stimulus: because of the nonlinearities, the dynamical state at the end of the RSI also depends on the coherence level.

Typical experiments on monkeys make use of reward-based protocols, hence with feedback. This makes difficult to have electrophysiological data in the absence of feedback. Yet, as discussed in this paper, the faster buildup of neural activity in post-correct trials than in post-error trials, as observed by Purcell and Kiani (2016) on monkeys in random-dot experiments, can be understood within our framework as a faster dynamics near the boundary between attraction basins in the post-correct case.

As discussed above, another prediction of the model is that, in the case of PES or PIA, the mean activity of the losing neural population is different for correct and error decisions, a difference which should correlate with the amplitude of the effect.

\section{First- and higher-order sequential effects}

Sequential effects can be categorized as first order (if caused by the immediately previous trial), or higher order (if caused by earlier trials in the sequence; Laming, 1979a; Soetens et al., 1984, 1985; Cho et al., 2002). Post-error adjustments have also been experimentally observed at higher order (Laming, 1979a).

Within the framework of attractor networks, the sequential effects in choice repetitions are explained by a starting bias, as discussed by Gao et al. (2009) and Bonaiuto et al. (2016), and in the present paper. As stated by Gao et al. (2009), without any additional memory module, an attractor network cannot reproduce the transition between automatic facilitation and strategic expectancy (Laming, 1968). In our network, for too short RSIs (a few dozens of milliseconds) the sequential effects are too strong to be plausible. Decision conflict mechanisms (Jones et al., 2002) could be implemented to correct this effect and to investigate other effects of repetitions and alternations (Gao et al., 2009).

To account for higher-order effects, Gao et al. (2009) considered a dynamical network making use of additional memory modules. This network is explicitly set up to reproduce automatic facilitation and strategic expectancy effects. In this model, even the first-order effects result from a coupling between a short-term memory module and the decision network. In contrast, we have shown here that a single attractor network, without memory units, presents first-order effects as an intrinsic property of the dynamics.

However, because of the nature of the dynamics in our model, we do not expect to reproduce higher-order effects. Indeed, for parameters for which the model exhibits first-order sequential effects $\left(I_{\mathrm{CD} \text {, } \max }=0.035 \mathrm{nA}\right)$, we find neither second-order sequential effects, nor post-error adjustments, as illustrated in Figure $13 C$ and $D$.

One may ask whether a more complex architecture, taking into account other brain areas, could account for higher-order repetition biases and post-error adjustments effects as resulting from some intrinsic properties of the dynamics, in the absence of specific memory units.

\section{Working memory and decision-making}

In this work we have considered free response time task (Roitman and Shadlen, 2002) in which the subject must make a decision as soon as possible. In the different protocol delayed visual motion discrimination experiment (Shadlen and Newsome, 2001), the subject must make the decision at a prescribed time after the onset of the stimulus. In such task, the decision choice must be stored to be retrieved at the prescribed instant of time. In the original attractor neural network model (Wang, 2002), the decision is stored as in a working memory. As discussed at the beginning of this paper, within the framework of a single module of attractor decision network, the $\mathrm{CD}$ considered in the present paper allows the system to make successive decisions, at the price of removing the working memory behavior. An important issue is to understand how the decisionmaking system can adapt itself to these opposite contexts (for a model with gain modulation, see Niyogi and Wong-Lin, 2013 ). It is not unrealistic to expect a control mechanism onto the inhibitory current. Depending on the task, the inhibitory current could be sent either just after the decision has been made, or later after the end of the delay period. In the latter case, a prediction is that, compared with cases without delay, there should be weaker post-error effects, but stronger repeated/alternated effects.

An alternative is to have a more complex architecture. However, the memory units used by Gao et al. (2009) are not appropriate for dealing with delayed discrimination experiments. For experiments with delays, Murray et al. (2017) consider two interacting modules, one implementing the posterior parietal cortex and another one the 
posterior frontal cortex. It will be interesting to extend the present work by adding a working memory module in line with Murray et al. (2017), to obtain a network performing sequential decision-making while keeping the working memory behavior.

Finally we note that various brain areas have been shown to be involved in sequential decision tasks in which the memory of the last decision has to be maintained (Middlebrooks and Sommer, 2012; Donahue et al., 2013; Abzug and Sommer, 2018). This suggests more generally that a broader network is necessary for decision tasks requiring memory.

\section{Future prospects}

During behavioral tasks, subjects are not always aware of their mistakes (Yeung and Summerfield, 2012), but do show PES. One may thus ask why one does not generally become aware that an error has been made, because the neural dynamics is different following an error or a success. As discussed in the present work, these differences in the dynamics are very subtle. The post-error and post-correct firing rates have broad distributions, with some common properties (the same mean for example). The strong overlapping of these distributions makes it difficult to infer the correctness of the decision on a single trial basis. Yet, the tails of the post-error synaptic distribution should allow in some cases to infer that an error has been made. It would be interesting to see in behavioral experiments whether the post-error effects can be related to the confidence in one's decision (Wei et al., 2015; Insabato et al., 2017).

\section{References}

Abbott LF, Chance FS (2005) Drivers and modulators from push-pull and balanced synaptic input. Prog Brain Res 149:147-155. CrossRef Medline

Abzug ZM, Sommer MA (2018) Serial decision-making in monkeys during an oculomotor task. J Exp Psychol Anim Learn Cogn 44:95-102. CrossRef Medline

Ashby F (1983) A biased random walk model for two choice reaction times. J Math Psychol 27:277-297. CrossRef

Beck JM, Ma WJ, Kiani R, Hanks T, Churchland AK, Roitman J, Shadlen MN, Latham PE, Pouget A (2008) Probabilistic population codes for Bayesian decision making. Neuron 60:1142-1152. CrossRef Medline

Benjamin DJ, Berger JO, Johannesson M, Nosek BA, Wagenmakers EJ, Berk R, Bollen KA, Brembs B, Brown L, Camerer C, Cesarini D, Chambers CD, Clyde M, Cook TD, De Boeck P, Dienes Z, Dreber A, Easwaran K, Efferson C, Fehr E, et al. (2018) Redefine statistical significance. Nat Hum Behav 2:6-10. CrossRef

Bezanson J, Edelman A, Karpinski S, Shah VB (2017) Julia: a fresh approach to numerical computing. SIAM Rev 59:65-98. CrossRef

Bliss DP, D’Esposito M (2017) Synaptic augmentation in a cortical circuit model reproduces serial dependence in visual working memory. PLoS One 12:e0188927. CrossRef Medline

Bogacz R, Brown E, Moehlis J, Holmes P, Cohen JD (2006) The physics of optimal decision making: a formal analysis of models of performance in two-alternative forced-choice tasks. Psychol Rev 113:700-765. CrossRef Medline

Bonaiuto JJ, De Berker A, Bestmann S (2016) Response repetition biases in human perceptual decisions are explained by activity decay in competitive attractor models. eLife 5:e20047. CrossRef Medline

Bonnasse-Gahot L, Nadal JP (2012) Perception of categories: from coding efficiency to reaction times. Brain Res 1434:47-61. CrossRef Medline

Busemeyer JR, Townsend JT (1993) Decision field theory: a dynamicscognitive approach to desion making in an uncertain environment. Psychol Rev 100:432-459. CrossRef Medline

Cho RY, Nystrom LE, Brown ET, Jones AD, Braver TS, Holmes PJ, Cohen JD (2002) Mechanisms underlying dependencies of performance on stimulus history in a two-alternative forced-choice task. Cogn Affect Behav Neurosci 2:283-299. CrossRef Medline

Cohen MX, van Gaal S, Ridderinkhof KR, Lamme VA (2009) Unconscious errors enhance prefrontal-occipital oscillatory synchrony. Front Hum Neurosci 3:54. Medline

Compte A, Brunel N, Goldman-Rakic PS, Wang XJ (2000) Synaptic mech- anisms and network dynamics underlying spatial working memory in a cortical network model. Cereb Cortex 10:910-923. CrossRef Medline

Crapse TB, Sommer MA (2008) Corollary discharges across the animal kingdom. Nat Rev Neurosci 9:587-600. CrossRef Medline

Crapse TB, Sommer MA (2009) Frontal eye field neurons with spatial representations predicted by their subcortical input. J Neurosci 29:53085318. CrossRef Medline

Danielmeier C, Ullsperger M (2011) Post-error adjustments. Front Psychol 2:233. CrossRef Medline

Danielmeier C, Eichele T, Forstmann BU, Tittgemeyer M, Ullsperger M (2011) Posterior medial frontal cortex activity predicts post-error adaptations in task-related visual and motor areas. J Neurosci 31:1780-1789. CrossRef Medline

Debener S, Ullsperger M, Siegel M, Fiehler K, von Cramon DY, Engel AK (2005) Trial-by-trial coupling of concurrent electroencephalogram and functional magnetic resonance imaging identifies the dynamics of performance monitoring. J Neurosci 25:11730-11737. CrossRef Medline

Deco G, Ponce-Alvarez A, Mantini D, Romani GL, Hagmann P, Corbetta M (2013) Resting-state functional connectivity emerges from structurally and dynamically shaped slow linear fluctuations. J Neurosci 33:1123911252. CrossRef Medline

Ding L, Hikosaka O (2006) Comparison of reward modulation in the frontal eye field and caudate of the macaque. J Neurosci 26:6695-6703. CrossRef Medline

Donahue CH, Seo H, Lee D (2013) Cortical signals for rewarded actions and strategic exploration. Neuron 80:223-234. CrossRef Medline

Dutilh G, Vandekerckhove J, Forstmann BU, Keuleers E, Brysbaert M, Wagenmakers EJ (2012) Testing theories of post-error slowing. Atten Percept Psychophys 74:454-465. CrossRef Medline

Efron B, Tibshirani RJ (1994) An introduction to the bootstrap. Boca Raton, FL: Chapman and Hall/CRC.

Engel TA, Wang XJ (2011) Same or different? A neural circuit mechanism of similarity-based pattern match decision making. J Neurosci 31:69826996. CrossRef Medline

Engel TA, Chaisangmongkon W, Freedman DJ, Wang XJ (2015) Choicecorrelated activity fluctuations underlie learning of neuronal category representation. Nat Commun 6:6454. CrossRef Medline

Ermentrout GB, Mahajan A (2003) Simulating, analyzing, and animating dynamical systems: a guide to XPPAUT for researchers and students, Vol 56. Philadelphia: SIAM.

Farrell S, Ludwig CJ (2008) Bayesian and maximum likelihood estimation of hierarchical response time models. Psychon Bull Rev 15:1209-1217. CrossRef Medline

Fecteau JH, Munoz DP (2003) Exploring the consequences of the previous trial. Nat Rev Neurosci 4:435-443. CrossRef Medline

Finkel AS, Redman SJ (1983) The synaptic current evoked in cat spinal motoneurones by impulses in single group 1a axons. J Physiol 342:615-632. CrossRef Medline

Fleming SM, Weil RS, Nagy Z, Dolan RJ, Rees G (2010) Relating introspective accuracy to individual differences in brain structure. Science 329: 1541-1543. CrossRef Medline

Freedman DJ, Riesenhuber M, Poggio T, Miller EK (2003) A comparison of primate prefrontal and inferior temporal cortices during visual categorization. J Neurosci 23:5235-5246. CrossRef Medline

Ganguli S, Bisley JW, Roitman JD, Shadlen MN, Goldberg ME, Miller KD (2008) One-dimensional dynamics of attention and decision making in LIP. Neuron 58:15-25. CrossRef Medline

Gao J, Wong-Lin K, Holmes P, Simen P, Cohen JD (2009) Sequential effects in two-choice reaction time tasks: decomposition and synthesis of mechanisms. Neural Comput 21:2407-2436. CrossRef Medline

Gehring WJ, Fencsik DE (2001) Functions of the medial frontal cortex in the processing of conflict and errors. J Neurosci 21:9430-9437. CrossRef Medline

Goldfarb S, Wong-Lin KF, Schwemmer M, Leonard NE, Holmes P (2012) Can post-error dynamics explain sequential reaction time patterns? Front Psychol 3:213. CrossRef Medline

Hall WC, Moschovakis AK (2003) The superior colliculus: new approaches for studying sensorimotor integration. Boca Raton, FL: CRC.

Hester R, Foxe JJ, Molholm S, Shpaner M, Garavan H (2005) Neural mechanisms involved in error processing: a comparison of errors made with and without awareness. Neuroimage 27:602-608. CrossRef Medline 
Hollander M, Wolfe DA, Chicken E (2014) Nonparametric statistical methods, Vol 2. Hoboken, NJ: Wiley.

Hsiao PY, Lo CC (2013) A plastic cortico-striatal circuit model of adaptation in perceptual decision. Front Comput Neurosci 7:178. CrossRef Medline

Insabato A, Pannunzi M, Deco G (2017) Multiple choice neurodynamical model of the uncertain option task. PLoS Comput Biol 13:e1005250. CrossRef Medline

Jentzsch I, Dudschig C (2009) Why do we slow down after an error? mechanisms underlying the effects of posterror slowing. QJ Exp Psychol 62: 209-218. CrossRef Medline

Jones AD, Cho RY, Nystrom LE, Cohen JD, Braver TS (2002) A computational model of anterior cingulate function in speeded response tasks: effects of frequency, sequence, and conflict. Cogn Affect Behav Neurosci 2:300-317. CrossRef Medline

King JA, Korb FM, von Cramon DY, Ullsperger M (2010) Post-error behavioral adjustments are facilitated by activation and suppression of taskrelevant and task-irrelevant information processing. J Neurosci 30: 12759-12769. CrossRef Medline

Laming D (1979a) Choice reaction performance following an error. Acta Psychologica 43:199-224. CrossRef

Laming D (1979b) Autocorrelation of choice-reaction times. Acta Psychologica 43:381-412. CrossRef Medline

Laming DR (1968) Information theory of choice-reaction times. Information theory of choice reaction times, p. 172. London: Academic.

Lauwereyns J, Watanabe K, Coe B, Hikosaka O (2002) A neural correlate of response bias in monkey caudate nucleus. Nature 418:413-417. CrossRef Medline

Liberman A, Harris K, Hoffman H, Griffith B (1957) The discrimination of speech sounds within and across phoneme boundaries. J Exp Psychol 54:358-368. CrossRef Medline

Lo CC, Wang XJ (2006) Cortico-basal ganglia circuit mechanism for a decision threshold in reaction time tasks. Nat Neurosci 9:956-963. CrossRef Medline

Marco-Pallarés J, Camara E, Münte TF, Rodríguez-Fornells A (2008) Neural mechanisms underlying adaptive actions after slips. J Cogn Neurosci 20:1595-1610. CrossRef Medline

Middlebrooks PG, Sommer MA (2012) Neuronal correlates of metacognition in primate frontal cortex paul. Neuron 75:517-530. CrossRef Medline

Miller P, Katz DB (2013) Accuracy and response-time distributions for decision-making: linear perfect integrators versus nonlinear attractor-based neural circuits. J Comput Neurosci 35:261-294. CrossRef Medline

Murray JD, Jaramillo J, Wang XJ (2017) Working memory and decision making in a frontoparietal circuit model. J Neurosci 37:12167-12186. CrossRef Medline

Niyogi RK, Wong-Lin K (2013) Dynamic excitatory and inhibitory gain modulation can produce flexible, robust and optimal decision-making. PLoS Comput Biol 9:e1003099. CrossRef Medline

Notebaert W, Houtman F, Opstal FV, Gevers W, Fias W, Verguts T (2009) Post-error slowing: an orienting account. Cognition 111:275-279. CrossRef Medline

Núñez Castellar E, Kühn S, Fias W, Notebaert W (2010) Outcome expectancy and not accuracy determines posterror slowing: ERP support. Cogn Affect Behav Neurosci 10:270-278. CrossRef Medline

Padoa-Schioppa C (2013) Neuronal origins of choice variability in economic decisions. Neuron 80:1322-1336. CrossRef Medline

Purcell BA, Kiani R (2016) Neural mechanisms of post-error adjustments of decision policy in parietal cortex. Neuron 89:658-671. CrossRef Medline

Quick RFJr (1974) A vector-magnitude model of contrast detection. Kybernetik 16:65-67. CrossRef Medline

Rabbitt P (2002) Consciousness is slower than you think. Q J Exp Psychol 55:1081-1092. CrossRef Medline

Rabbitt P, Rodgers B (1977) What does a man do after he makes an error? an analysis of response programming. Q J Exp Psychol 29:727-743. CrossRef

Rao V, DeAngelis GC, Snyder LH (2012) Neural correlates of prior expectations of motion in the lateral intraparietal and middle temporal areas. J Neurosci 32:10063-10074. CrossRef Medline

Ratcliff R (1978) A theory of memory retrieval. Psychol Rev 85:59-108. CrossRef
Ratcliff R, Smith PL (2004) A comparison of sequential sampling models for two-choice reaction time. Psychol Rev 111:333-367. CrossRef Medline

Ratcliff R, McKoon G (2008) The diffusion decision model: theory and data for two-choice decision tasks. Neural Comput 20:873-922. CrossRef Medline

Rizzo ML, Székely GJ (2016) Energy distance. WIREs Comput Stat 8:27-38. CrossRef

Rizzo M, Székely G (2014) Energy: E-statistics (energy statistics). R package version 1.6.2.

Rodriguez-Fornells A, Kurzbuch AR, Münte TF (2002) Time course of error detection and correction in humans: neurophysiological evidence. J Neurosci 22:9990-9996. CrossRef Medline

Roitman JD, Shadlen MN (2002) Response of neurons in the lateral intraparietal area during a combined visual discrimination reaction time task. J Neurosci 22:9475-9489. CrossRef Medline

Saito Y, Isa T (2003) Local excitatory network and NMDA receptor activation generate a synchronous and bursting command from the superior colliculus. J Neurosci 23:5854-5864. CrossRef Medline

Scudder CA, Kaneko CS, Fuchs AF (2002) The brainstem burst generator for saccadic eye movements: a modern synthesis. Exp Brain Res 142:439_ 462. CrossRef Medline

Shadlen MN, Newsome WT (1996) Motion perception: seeing and deciding. Proc Natl Acad Sci U S A 93:628-633. CrossRef Medline

Shadlen MN, Newsome WT (2001) Neural basis of a perceptual decision in the parietal cortex (area lip) of the rhesus monkey. J Neurophysiol 86: 1916-1936. CrossRef Medline

Shadlen MN, Hanks TD, Churchland AK, Kiani R, Yang T (2006) The speed and accuracy of a simple perceptual decision: a mathematical primer. In: Bayesian brain: probabilistic approaches to neural coding, pp 207-233. Boston: MIT.

Shorack GR, Wellner JA (2009) Empirical processes with applications to statistics. Philadelphia: SIAM.

Soetens E, Deboeck M, Hueting J (1984) Automatic aftereffects in twochoice reaction time: a mathematical representation of some concepts. J Exp Psychol Hum Percept Perform 10:581-598. CrossRef Medline

Soetens E, Boer LC, Hueting JE (1985) Expectancy or automatic facilitation? separating sequential effects in two-choice reaction time. J Exp Psychol Hum Percept Perform 11:598-616. CrossRef

Sommer MA, Wurtz RH (2002) A pathway in primate brain for internal monitoring of movements. Science 296:1480-1482. CrossRef Medline

Sommer MA, Wurtz RH (2008) Visual perception and corollary discharge. Perception 37:408-418. CrossRef Medline

Szekely G, Rizzo M (2013) Energy statistics: statistics based on distances. J Stat Plan Inference 143:1249-1272. CrossRef

Townsend JT, Ashby FG (1983) The stochastic modeling of elementary psychological processes. Cambridge, UK: Cambridge UP.

Usher M, McClelland JL (2001) The time course of perceptual choice: the leaky, competing accumulator model. Psychol Rev 108:550-592. CrossRef Medline

Verguts T, Notebaert W, Kunde W, Wühr P (2011) Post-conflict slowing: cognitive adaptation after conflict processing. Psychon Bull Rev 18:7682. CrossRef Medline

Vickers D (1979) Decision processes in Visual Perception. New York: Academic.

Wang XJ (2002) Probabilistic decision making by slow reverberation in cortical circuits. Neuron 36:955-968. CrossRef Medline

Wei W, Rubin JE, Wang XJ (2015) Role of the indirect pathway of the basal ganglia in perceptual decision making. J Neurosci 35:4052-4064. CrossRef Medline

Wong KF, Wang XJ (2006) A recurrent network mechanism of time integration in perceptual decisions. J Neurosci 26:1314-1328. CrossRef Medline

Wong KF, Huk AC, Shadlen MN, Wang XJ (2007) Neural circuit dynamics underlying accumulation of time-varying evidence during perceptual decision making. Front Comput Neurosci 1:6. CrossRef Medline

Yang Y, Cao P, Yang Y, Wang SR (2008) Corollary discharge circuits for saccadic modulation of the pigeon visual system. Nat Neurosci 11:595602. CrossRef Medline

Yeung N, Summerfield C (2012) Metacognition in human decision-making: confidence and error monitoring. Philos Trans R Soc Lond B Biol Sci 367:1310-1321. CrossRef Medline 